\title{
Model for Vaccine Design by Prediction of B-Epitopes of IEDB Given Perturbations in Peptide Sequence, In Vivo Process, Experimental Techniques, and Source or Host Organisms
}

\author{
Humberto González-Díaz, ${ }^{1,2}$ Lázaro G. Pérez-Montoto, ${ }^{3}$ and Florencio M. Ubeira ${ }^{3}$ \\ ${ }^{1}$ Department of Organic Chemistry II, University of the Basque Country UPV/EHU, 48940 Bilbao, Spain \\ ${ }^{2}$ Ikerbasque, Basque Foundation for Science, 48011 Bilbao, Spain \\ ${ }^{3}$ Department of Microbiology and Parasitology, University of Santiago de Compostela (USC), 15782 Santiago de Compostela, Spain
}

Correspondence should be addressed to Humberto González-Díaz; humberto.gonzalezdiaz@ehu.es

Received 21 August 2013; Accepted 17 November 2013; Published 12 January 2014

Academic Editor: Darren R. Flower

Copyright (C) 2014 Humberto González-Díaz et al. This is an open access article distributed under the Creative Commons Attribution License, which permits unrestricted use, distribution, and reproduction in any medium, provided the original work is properly cited.

\begin{abstract}
Perturbation methods add variation terms to a known experimental solution of one problem to approach a solution for a related problem without known exact solution. One problem of this type in immunology is the prediction of the possible action of epitope of one peptide after a perturbation or variation in the structure of a known peptide and/or other boundary conditions (host organism, biological process, and experimental assay). However, to the best of our knowledge, there are no reports of general-purpose perturbation models to solve this problem. In a recent work, we introduced a new quantitative structure-property relationship theory for the study of perturbations in complex biomolecular systems. In this work, we developed the first model able to classify more than 200,000 cases of perturbations with accuracy, sensitivity, and specificity $>90 \%$ both in training and validation series. The perturbations include structural changes in $>50000$ peptides determined in experimental assays with boundary conditions involving $>500$ source organisms, $>50$ host organisms, $>10$ biological process, and $>30$ experimental techniques. The model may be useful for the prediction of new epitopes or the optimization of known peptides towards computational vaccine design.
\end{abstract}

\section{Introduction}

National Institute of Allergy and Infectious Diseases (NIAID) supported the launch, in 2004, of the Immune Epitope Database (IEDB), http://www.iedb.org/ [1-4]. The IEDB system withdrew information from approximately $99 \%$ of all papers published to date that describe immune epitopes. In doing so, IEDB system analyses over 22 million PubMed abstracts and subsequently curated $\approx 13 \mathrm{~K}$ references, including $\approx 7 \mathrm{~K}$ manuscripts about infectious diseases, $\approx 1 \mathrm{~K}$ about allergy topics, $\approx 4 \mathrm{~K}$ about autoimmunity, and $1 \mathrm{~K}$ about transplant/alloantigen topics [5]. IEDB lists a huge amount of information about the molecular structure as well as the experimental conditions $\left(c_{i j}\right)$ in which different $i$ th molecules were determined to be immune epitopes or not. This explosion of information makes necessary both query/display functions for retrieval of known data from IEDB as well predictive tools for new epitopes. Salimi et al. [5] reviewed advances in epitope analysis and predictive tools available in the IEDB. In fact, IEDB analysis resource (IEDB-AR: http://tools.iedb.org/) is a collection of tools for prediction of molecular targets of Tand B-cell immune responses (i.e., epitopes) $[6,7]$.

On the other hand, Quantitative Structure-Activity/Property Relationships (QSAR/QSPR) techniques are useful tool to predict new drugs, RNA, drug-protein complexes, and protein-protein complexes. In general, QSAR/QSPR-like methods transform molecular structures into numeric molecular descriptors $\left(\lambda_{i}\right)$ in a first stage and later fit a model to predict the biological process. For example, DRAGON [8-10], CODESSA [11, 12], MOE [13], TOPS-MODE [14-17], TOMOCOMD $[18,19]$, and MARCH-INSIDE [20] are among the most used softwares to calculate molecular descriptors based on quantum mechanics (QM) and/or graph theory [21-27]. The software STATISTICA [28] and WEKA [29] are often 
used to perform multivariate statistics and/or machine learning (ML) analysis in order to preprocess data and later fit the final QSAR/QSPR model using techniques like principal component analysis (PCA), linear discriminant analysis (LDA), support vector machine (SVM), or artificial neural networks (ANN) [28].

QSAR/QSPR models are also important in immunoinformatics to predict the propensity of different molecular structures to play different roles in immunological processes. They include skin vaccine adjuvants and sensitizers [30-38], drugs and their activity/toxicity protein targets in the immune system [39], and epitopes [40-49]. Moreover, Reche and Reinherz [50] implemented PEPVAC (promiscuous epitopebased vaccine), a web server for the formulation of multiepitope vaccines that predict peptides binding to five distinct HLA class I supertypes (A2, A3, B7, A24, and B15). PEPVAC can also identify conserved MHC ligands, as well as those with a C-terminus resulting from proteasomal cleavage. The Dana-Farber Cancer Institute hosted the PEPVAC server at the site http://immunax.dfci.harvard.edu/PEPVAC/. To close with a last example, Lafuente and Reche [51] reviewed the available methods for predicting MHC-peptide binding and discussed their most relevant advantages and drawbacks.

In many complex QSPR-like problems in immunoinformatics, like in other areas, we know the exact experimental result (known solution) of the problem, but we are interested in the possible result obtained after a change (perturbation) on one or multiple values of the initial conditions of the experiment (new solution). For instance, we often know, for large collections of $i$ th molecules $\left(m_{i}\right)$, organic compounds, drugs, xenobiotics, and/or peptide sequences, the efficiency of the compound $\varepsilon\left(c_{i j}\right)$ as adjuvant, action as epitope, immunotoxicity, and/or the interaction (affinity, inhibition, etc.) with immunological targets. In addition, we often known for each molecule the exact conditions $\left(c_{i j}\right)$ of assay for the initial experiment including structure of the molecule $m_{i}$ (drug, adjuvant, and sequence of the peptide), source organism (so), host organism (ho), immunological process (ip), experimental technique (tq), concentration, temperature, time, solvents, and coadjuvants. This is the case of big data retrieved from very large databases like IEDB [1-4] and CHEMBL [52]. However, we do not know the possible result of the experiment if we change at least one of these conditions (perturbation). We refer to small changes or perturbations in both structure and condition for input or output variables. It means that we include changes in ho, so, ip, and tq, changes of the compound by one analogue compound with similar structure, changes in the sequence of the epitope (artificial by organic synthesis or natural mutations), and polarity of the solvent or coadjuvants. In these cases, we could use a perturbation theory model to solve the QSAR/QSPR problem. Perturbation theory includes methods that add "small" terms to a known solution of a problem in order to approach a solution to a related problem without known solution. Perturbation models have been widely used in all branches of science from QM to astronomy and life sciences including chaos or "butterfly effect," Bohr's atomic theory, Heisenberg's mechanics, Zeeman's and Stark's effects, and other models with applications in like protein spectroscopy and others [5357]. In a very recent work Gonzalez-Diaz et al. [58] formulated a general-purpose perturbation theory or model for multiple-boundary QSPR/QSAR problems. However, there is not report in the immunoinformatics literature of a general QSPR perturbation model for IEDB B-epitopes. Here we report the first example of QSPR-perturbation model for Bepitopes reported in IEDB able to predict the probability of occurrence of an epitope after a perturbation in the sequence, the experimental technique, the exposition process, and/or the source or host organisms.

\section{Materials and Methods}

2.1. Molecular Descriptors for Peptides. We calculated the molecular descriptors of the structure of peptides using the software MARCH-INSIDE (MI) based on the algorithm with the same name [59]. The MI approach uses a Markov Chain method to calculate the $k$ th mean values of different physicochemical molecular properties $\lambda\left(m_{i}\right)$ for $i$ th molecules $(m)$. These $\lambda\left(m_{i}\right)$ values are calculated as an average of ${ }^{k} \lambda\left(m_{i}\right)$ values for all atoms placed at topological distance $d \leq k$; which are in turn the means of atomic properties $\left(\lambda_{j}\right)$ for all atoms in the molecule and its neighbors placed at $d=k$. For instance, it is possible to derive average estimations of molecular refractivities ${ }^{k} \mathrm{MR}\left(m_{i}\right)$, partition coefficients ${ }^{k} P\left(m_{i}\right)$, and hardness ${ }^{k} \eta\left(m_{i}\right)$ for atoms placed at different topological distances $d \leq k$. In this first work, we calculated only one type of $\lambda\left(m_{i}\right)$ values. We calculated for all peptides the average value $\chi\left(m_{i}\right)$ of all the atomic electronegativities $\chi_{i}$ for all $\delta_{i}$ atoms connected to the $i$ th atom $(i \rightarrow j)$ and their neighbors placed at a distance $d \leq 5$ [59]:

$$
\chi\left(m_{i}\right)=\frac{1}{6} \sum_{k=0}^{5}{ }^{k} \chi_{j}=\frac{1}{6} \sum_{k=0}^{5} \sum_{i \rightarrow j}^{\delta_{i}} p_{k}\left(\chi_{j}\right) \cdot \chi_{j} .
$$

We calculate the probabilities ${ }^{k} p\left(\lambda_{j}\right)$ for any atomic property including ${ }^{k} p\left(\chi_{j}\right)$ using a Markov Chain model for the gradual effects of the neighboring atoms at different distances in the molecular backbone. This method has been explained in detail in many previous works so we omit the details here [59].

2.2. Electronegativity Perturbation Model for Prediction of BEpitopes. Very recently Gonzalez-Diaz et al. [58] formulated a general-purpose perturbation theory or model for multipleboundary QSPR/QSAR problems. We adapted here this new theory or modeling method to approach to the peptide prediction problem from the point of view of perturbation theory. Let be a set of $i$ th peptide molecules denoted as $m_{i}$ with a value of efficiency $\varepsilon_{i j}$ as epitopes experimentally determined under a set of boundary conditions $c_{j} \equiv\left(c_{0}, c_{1}, c_{2}, c_{3}, \ldots, c_{n}\right)$. We put the main emphasis here on peptides reported in the database IEDB. In this sense, the boundary conditions $c_{j}$ used here are the same reported in this database, $c_{0}=$ is the specific 
peptide, $c_{1}=\mathrm{so}_{j}, c_{2}=\mathrm{ho}_{j}, c_{3}=\mathrm{ip}_{j}$, and $c_{4}=\mathrm{tq}_{j}$. In general, so is the organism that expresses the peptide (but it can include also artificial peptides, cellular lines, etc.), ho is the host organism exposed to the peptide by means of the bp detected with tq. As our analysis, based on the data reported by IEDB we are unable to work with continuous values of epitope activity $\varepsilon_{i j}$. Consequently, we have to predict the discrete function of B-epitope efficiency $\lambda\left(\varepsilon_{i j}\right)=1$ for epitopes reported in the conditions $c_{j}$ and $\lambda\left(\varepsilon_{i j}\right)=0$, otherwise. Our main aim is to predict the shift or change in a function of the output efficiency $\Delta \lambda\left(\varepsilon_{i j}\right)=\lambda\left(\varepsilon_{i j}\right)_{\text {ref }}-\lambda\left(\varepsilon_{i j}\right)_{\text {new }}$ that takes place after a change, variation, or perturbation $(\Delta V)$ in the structure and/or boundary conditions of a peptide of reference. But we know the efficiency of the process of reference $\lambda\left(\varepsilon_{i j}\right)_{\text {ref }}$ in addition to the molecular structure and the set of conditions $c_{j}$ for initial (reference) and final processes (new). Consequently, to predict $\Delta \lambda\left(\varepsilon_{i j}\right)$ we have to predict only $\lambda\left(\varepsilon_{i j}\right)_{\text {new }}$ the efficiency function of the new state obtained by a change in the structure of the peptide and/or the boundary conditions. Let $\Delta V$ be a perturbation in a function $\lambda$; we can define $V_{i j}$ as the state information function for the reference and new states. According to our recent model [58], we can write $V_{i j}$ as a function of the conditions and structure of the peptide $m_{i}$ as follows. In fact, the variational state functions $V_{i j}$ have to be written in pairs in order to describe the initial (reference) and final (new) states of a perturbation, as follow:

$$
\begin{gathered}
V_{i j}=\lambda\left(\varepsilon_{i j}\right)_{\mathrm{new}}-\sum_{j=1}^{4}\left(\lambda\left(m_{i}\right)-\lambda\left(c_{i j}\right)_{\mathrm{avg}}\right), \\
V_{q r}=\lambda\left(\varepsilon_{q r}\right)_{\mathrm{ref}}-\sum_{r=1}^{4}\left(\lambda\left(m_{q}\right)-\lambda\left(c_{q r}\right)_{\mathrm{avg}}\right) .
\end{gathered}
$$

The state function ${ }^{n} V_{i j}$ is for the $i$ th peptide measured under a set of $c_{i j}$ boundary conditions in output, final, or new state. The conjugated state function ${ }^{r} V_{q r}$ is for the $q$ th peptide measured under a set of $c_{q r}$ boundary conditions for the input, initial, or reference state. The difference $\Delta V$ between the new (output) state and the reference (input) state is the additive perturbation [58]. Consider

$$
\begin{aligned}
\Delta V=V_{i j}-V_{q r}= & {\left[\lambda\left(\varepsilon_{i j}\right)_{\text {new }}-\sum_{j=1}^{4}\left(\lambda\left(m_{i}\right)-\lambda\left(c_{i j}\right)_{\mathrm{avg}}\right)\right] } \\
& -\left[\lambda\left(\varepsilon_{q r}\right)_{\mathrm{ref}}-\sum_{r=1}^{4}\left(\lambda\left(m_{q}\right)-\lambda\left(c_{q r}\right)_{\mathrm{avg}}\right)\right] .
\end{aligned}
$$

Equation (3) described before opens the door to test different hypothesis. A simple hypotheses is $\mathrm{H}_{0}$ : existence of one small and constant value of the perturbation function $\Delta V=e_{0}$ for all the pairs of peptides and a linear relationship
TABLE 1: Results of QSPR-perturbation model for IEDB B-Epitopes.

\begin{tabular}{lcccc}
\hline Data & Stat. & Pred. & \multicolumn{2}{c}{ Predicted epitope perturbations } \\
subset & param. & $\%$ & $\lambda\left(\varepsilon_{i j}\right)=1$ & $\lambda\left(\varepsilon_{i j}\right)=0$ \\
\hline$\lambda\left(\varepsilon_{i j}\right)=1$ & Sp & 97.0 & $\mathbf{8 4 6 0 7}$ & 2660 \\
$\lambda\left(\varepsilon_{i j}\right)=0$ & Sn & 93.6 & 4354 & $\mathbf{6 3 5 4 8}$ \\
\hline Total train & Ac & 95.5 & & \\
\hline$\lambda\left(\varepsilon_{i j}\right)=1$ & Sp & 97.1 & $\mathbf{2 8 0 6 0}$ & 840 \\
$\lambda\left(\varepsilon_{i j}\right)=0$ & Sn & 93.3 & 1485 & $\mathbf{2 0 6 4 1}$ \\
\hline Total cv & Ac & 95.4 & &
\end{tabular}

Bold font is used to highlight the number of cases correctly classified by the model.

between perturbations of input/output boundary conditions with coefficients $a_{i j}, b_{i j}, c_{q r}$, and $d_{i j}$. Consider

$$
\begin{aligned}
e_{0}= & \Delta V \\
= & {\left[a_{i j} \cdot \lambda\left(\varepsilon_{i j}\right)_{\text {new }}-\sum_{j=1}^{4} b_{i j} \cdot\left(\lambda\left(m_{i}\right)-\lambda\left(c_{j}\right)_{\text {avg }}\right)\right] } \\
& -\left[c_{q r} \cdot \lambda\left(\varepsilon_{q r}\right)_{\text {ref }}-\sum_{r=1}^{4} d_{q r} \cdot\left(\lambda\left(m_{q}\right)-\lambda\left(c_{r}\right)_{\text {avg }}\right)\right] .
\end{aligned}
$$

We can use elemental algebraic operations to obtain from these equations an expression for efficiency as epitope of the peptide $\lambda\left(\varepsilon_{i j}\right)_{\text {new }}$. In this case, considering $b_{i j} \approx d_{q r}$, we can obtain the different expressions; the last may be very useful to solve the QSRR problem for the large datasets formed by IEDB B-epitopes. Consider

$$
\begin{aligned}
\lambda\left(\varepsilon_{i j}\right)_{\text {new }}= & \left(\frac{c_{q r}}{a_{i j}}\right) \cdot \lambda\left(\varepsilon_{q r}\right)_{\text {ref }} \\
& +\left[\sum_{j=1}^{4}\left(\frac{b_{q r}}{a_{i j}}\right) \cdot\left(\lambda\left(m_{i}\right)-\lambda\left(c_{j}\right)_{\mathrm{avg}}\right)_{\mathrm{new}}\right] \\
& -\left[\sum_{r=1}^{4}\left(\frac{d_{q r}}{a_{i j}}\right) \cdot\left(\lambda\left(m_{q}\right)-\lambda\left(c_{r}\right)_{\mathrm{avg}}\right)_{\mathrm{ref}}\right] \\
& +\left(\frac{e_{0}}{a_{i j}}\right), \\
= & { }^{\prime} c_{0} \cdot \lambda\left(\varepsilon_{q r}\right)_{\mathrm{ref}} \\
& +\sum_{j=1}^{4}{ }^{\prime} d_{i j} \cdot \Delta\left(\lambda\left(m_{i j}\right)-\lambda\left(c_{j}\right)_{\mathrm{avg}}\right)+{ }^{\prime} e_{0}, \\
\lambda\left(\varepsilon_{i j}\right)_{\mathrm{new}}= & { }^{\prime} c_{0} \cdot \lambda\left(\varepsilon_{q r}\right)_{\mathrm{ref}}+\sum_{j=1}^{4}{ }^{\prime} d_{i j} \cdot \Delta \Delta \lambda_{i j q r}+{ }^{\prime} e_{0}, \\
\lambda\left(\varepsilon_{i j}\right)_{\text {new }}= & { }^{\prime} c_{0} \cdot \lambda\left(\varepsilon_{q r}\right)_{\mathrm{ref}}+\sum_{j=1}^{4}{ }^{\prime} d_{i j} \cdot \Delta \Delta \chi_{i j q r}+{ }^{\prime} e_{0} .
\end{aligned}
$$


TABLE 2: Average values and count of input-output cases for different organisms, process, and techniques.

\begin{tabular}{|c|c|c|c|}
\hline Source organism (so) & $N_{\text {in }}$ & $N_{\text {out }}$ & ${ }^{*} \chi$ \\
\hline Homo sapiens & 38920 & 39274 & 2.685 \\
\hline Plasmodium falciparum & 10669 & 9446 & 2.704 \\
\hline Hepatitis $C$ virus & 9935 & 10239 & 2.683 \\
\hline Bos taurus & 5671 & 5780 & 2.690 \\
\hline Canine parvovirus & 5655 & 5637 & 2.693 \\
\hline Foot-mouth disease virus & 4062 & 4176 & 2.676 \\
\hline Triticum aestivum & 3769 & 3887 & 2.703 \\
\hline Bacillus anthracis & 3602 & 3600 & 2.699 \\
\hline Human papillomavirus & 3316 & 3414 & 2.693 \\
\hline Human herpesvirus & 3026 & 3132 & 2.684 \\
\hline Gallus gallus & 2850 & 2829 & 2.689 \\
\hline Arachis hypogaea & 2648 & 2670 & 2.687 \\
\hline Mycobacterium tuberculosis & 2637 & 2593 & 2.688 \\
\hline Clostridium botulinum & 2588 & 2722 & 2.685 \\
\hline SARS coronavirus & 2550 & 2704 & 2.686 \\
\hline Mus musculus & 2334 & 2287 & 2.682 \\
\hline Hepatitis B virus & 2007 & 2066 & 2.680 \\
\hline Helicobacter pylori & 1958 & 1796 & 2.695 \\
\hline Hevea brasiliensis & 1938 & 1958 & 2.697 \\
\hline Hepatitis E virus & 1928 & 1941 & 2.685 \\
\hline Shigella flexneri & 1878 & 1701 & 2.699 \\
\hline Dengue virus 2 & 1767 & 1828 & 2.679 \\
\hline Staphylococcus aureus & 1757 & 1661 & 2.694 \\
\hline Treponema pallidum & 1739 & 1755 & 2.691 \\
\hline Escherichia coli & 1721 & 1678 & 2.689 \\
\hline Murine hepatitis virus & 1575 & 1603 & 2.692 \\
\hline Haemophilus influenzae & 1545 & 1587 & 2.695 \\
\hline Streptococcus mutans & 1523 & 1537 & 2.697 \\
\hline Puumala virus (strain) & 1505 & 1574 & 2.689 \\
\hline Chlamydia trachomatis & 1402 & 1546 & 2.704 \\
\hline Human respiratory virus & 1347 & 1398 & 2.682 \\
\hline Borrelia burgdorferi & 1228 & 1237 & 2.698 \\
\hline Hepatitis delta virus & 1182 & 1199 & 2.690 \\
\hline Streptococcus pyogenes & 1181 & 1251 & 2.697 \\
\hline Porphyromonas gingivalis & 1143 & 1085 & 2.688 \\
\hline Human enterovirus & 1106 & 1132 & 2.689 \\
\hline Influenza $A$ virus & 1085 & 1086 & 2.687 \\
\hline Mycoplasma hyopneumoniae & 1044 & 1024 & 2.695 \\
\hline Rattus norvegicus & 1025 & 1039 & 2.689 \\
\hline Bordetella pertussis & 1011 & 960 & 2.685 \\
\hline Human T-lymphotropic virus & 996 & 1031 & 2.680 \\
\hline Anaplasma marginale & 977 & 857 & 2.707 \\
\hline Measles virus strain & 804 & 810 & 2.688 \\
\hline Fasciola hepatica & 803 & 857 & 2.685 \\
\hline Neisseria meningitidis & 789 & 853 & 2.696 \\
\hline Human poliovirus & 766 & 780 & 2.690 \\
\hline Tityus serrulatus & 764 & 775 & 2.680 \\
\hline Torpedo californica & 752 & 788 & 2.687 \\
\hline
\end{tabular}

TABLE 2: Continued.

\begin{tabular}{|c|c|c|c|}
\hline Source organism (so) & $N_{\text {in }}$ & $N_{\text {out }}$ & ${ }^{*} \chi$ \\
\hline Cryptomeria japonica & 719 & 794 & 2.680 \\
\hline Mycobacterium bovis & 717 & 733 & 2.688 \\
\hline Trypanosoma cruzi & 691 & 777 & 2.704 \\
\hline Andes virus CHI-7913 & 679 & 687 & 2.690 \\
\hline Bovine papillomavirus & 672 & 665 & 2.692 \\
\hline Human hepatitis & 670 & 696 & 2.688 \\
\hline Leishmania infantum & 659 & 735 & 2.688 \\
\hline Human parvovirus & 649 & 691 & 2.683 \\
\hline Poa pratensis & 648 & 664 & 2.692 \\
\hline Aspergillus fumigatus & 642 & 709 & 2.677 \\
\hline Duck hepatitis & 587 & 603 & 2.688 \\
\hline Olea europaea & 571 & 577 & 2.692 \\
\hline Porcine reproductive & 515 & 514 & 2.681 \\
\hline Fagopyrum esculentum & 509 & 497 & 2.685 \\
\hline Juniperus ashei & 505 & 568 & 2.672 \\
\hline Mycobacterium leprae & 489 & 542 & 2.690 \\
\hline Glycine max & 477 & 509 & 2.685 \\
\hline D. pteronyssinus & 455 & 464 & 2.680 \\
\hline Plasmodium vivax & 453 & 446 & 2.690 \\
\hline Chlamydophila pneumoniae & 446 & 462 & 2.690 \\
\hline Pseudomonas aeruginosa & 443 & 454 & 2.691 \\
\hline Vibrio cholera & 427 & 426 & 2.694 \\
\hline Streptococcus sp. & 426 & 425 & 2.691 \\
\hline Mycobacterium avium & 425 & 415 & 2.689 \\
\hline Dermatophagoides farinae & 410 & 390 & 2.693 \\
\hline Human coxsackievirus & 406 & 392 & 2.694 \\
\hline Equine infectious virus & 404 & 419 & 2.688 \\
\hline Babesia equi & 383 & 371 & 2.696 \\
\hline Prunus dulcis & 383 & 379 & 2.708 \\
\hline Human adenovirus & 375 & 405 & 2.686 \\
\hline Theileria parva & 366 & 371 & 2.713 \\
\hline Candida albicans & 365 & 370 & 2.690 \\
\hline Porcine endogenous & 355 & 351 & 2.692 \\
\hline Ovis aries & 352 & 350 & 2.683 \\
\hline Chironomus thummi & 347 & 338 & 2.691 \\
\hline Sus scrofa & 343 & 362 & 2.686 \\
\hline Bovine leukemia virus & 333 & 329 & 2.676 \\
\hline Ricinus communis & 329 & 314 & 2.692 \\
\hline Androctonus australis & 322 & 357 & 2.685 \\
\hline Renibacterium salmoninarum & 319 & 350 & 2.690 \\
\hline Orientia tsutsugamushi & 309 & 372 & 2.705 \\
\hline Anacardium occidentale & 293 & 306 & 2.693 \\
\hline Conus geographus & 289 & 295 & 2.660 \\
\hline Host organism (ho) & $N_{\text {in }}$ & $N_{\text {out }}$ & ${ }^{*} \chi$ \\
\hline Homo sapiens & 257293 & 91093 & 2.6856 \\
\hline Mus musculus & 107867 & 51466 & 2.6873 \\
\hline Oryctolagus cuniculus & 65053 & 31433 & 2.6900 \\
\hline Bos taurus & 15333 & 2072 & 2.6909 \\
\hline Rattus norvegicus & 9450 & 3562 & 2.6876 \\
\hline Aotus sp. & 9044 & 3933 & 2.6879 \\
\hline Sus scrofa & 7725 & 3464 & 2.6873 \\
\hline Gallus gallus & 7507 & 997 & 2.6790 \\
\hline
\end{tabular}


TABLE 2: Continued.

\begin{tabular}{|c|c|c|c|}
\hline Source organism (so) & $N_{\text {in }}$ & $N_{\text {out }}$ & ${ }^{*} \chi$ \\
\hline Canis lupus & 6604 & 3334 & 2.6906 \\
\hline Macaca mulatta & 5261 & 2569 & 2.6993 \\
\hline Ovis aries & 3953 & 1653 & 2.6836 \\
\hline Equus caballus & 3943 & 2099 & 2.6842 \\
\hline Cavia porcellus & 3458 & 1688 & 2.6833 \\
\hline Capra hircus & 2182 & 1127 & 2.6830 \\
\hline Aotus nancymaae & 1659 & 852 & 2.6837 \\
\hline Pan troglodytes & 1614 & 732 & 2.6757 \\
\hline Marmota monax & 1100 & 509 & 2.7011 \\
\hline Felis catus & 901 & 279 & 2.6838 \\
\hline Myodes glareolus & 814 & 388 & 2.6863 \\
\hline Anas platyrhynchos & 688 & 342 & 2.6880 \\
\hline Homo sapiens (human) & 508 & 270 & 2.6851 \\
\hline Trichosurus vulpecula & 456 & 126 & 2.6921 \\
\hline Mesocricetus auratus & 438 & 104 & 2.6909 \\
\hline Macaca cyclopis & 382 & 193 & 2.6871 \\
\hline O. tshawytscha & 333 & 159 & 2.6929 \\
\hline Macaca fuscata & 188 & 100 & 2.6667 \\
\hline Cricetulus migratorius & 171 & 142 & 2.7008 \\
\hline Camelus dromedarius & 171 & 89 & 2.6886 \\
\hline Dicentrarchus labrax & 121 & 55 & 2.6759 \\
\hline Macaca fascicularis & 96 & 52 & 2.6793 \\
\hline Saimiri sciureus & 92 & 44 & 2.6900 \\
\hline Canis familiaris & 77 & 42 & 2.6850 \\
\hline Rattus rattus & 72 & 31 & 2.6760 \\
\hline Callithrix pygmaea & 67 & 30 & 2.6920 \\
\hline Chinchilla lanigera & 41 & 24 & 2.6729 \\
\hline Aotus lemurinus & 30 & 19 & 2.6860 \\
\hline Papio cynocephalus & 27 & 13 & 2.7267 \\
\hline Aotus griseimembra & 26 & 12 & 2.7000 \\
\hline Mustela vison & 18 & 10 & 2.7000 \\
\hline Chlorocebus aethiops & 15 & 10 & 2.6875 \\
\hline Bos indicus & 13 & 4 & 2.6925 \\
\hline Oncorhynchus mykiss & 10 & 4 & 2.6700 \\
\hline M. macquariensis & 9 & 6 & 2.6600 \\
\hline Cricetulus griseus & 8 & 4 & 2.6900 \\
\hline Aotus trivirgatus & 7 & 4 & 2.7000 \\
\hline Process type (pt) & $N_{\text {in }}$ & $N_{\text {out }}$ & ${ }^{*} \chi$ \\
\hline AID & 111197 & 108536 & 2.6876 \\
\hline OOID & 32419 & 32617 & 2.6868 \\
\hline OAID & 19210 & 18954 & 2.6801 \\
\hline OOA & 15863 & 16303 & 2.6902 \\
\hline NI & 13430 & 15206 & 2.6845 \\
\hline EWEIR & 4818 & 4864 & 2.6843 \\
\hline EEE & 3113 & 3546 & 2.6906 \\
\hline OOD & 2806 & 2799 & 2.6887 \\
\hline AICD & 1077 & 1095 & 2.6812 \\
\hline EWED & 696 & 686 & 2.6879 \\
\hline DEWED & 280 & 337 & 2.6804 \\
\hline $\mathrm{TT}$ & 260 & 215 & 2.6806 \\
\hline OOC & 153 & 137 & 2.6800 \\
\hline
\end{tabular}

TABLE 2: Continued.

\begin{tabular}{|c|c|c|c|}
\hline Source organism (so) & $N_{\text {in }}$ & $N_{\text {out }}$ & ${ }^{*} \chi$ \\
\hline Technique (tq) & $N_{\text {in }}$ & $N_{\text {out }}$ & ${ }^{*} \chi$ \\
\hline ELISA & 133458 & 135109 & 2.6871 \\
\hline WI & 33627 & 33292 & 2.6887 \\
\hline ACAbB & 7780 & 9068 & 2.6862 \\
\hline PhDIP & 7450 & 4496 & 2.6771 \\
\hline RIA & 5241 & 5218 & 2.6858 \\
\hline IFAIH & 4454 & 4581 & 2.6879 \\
\hline NIAA & 4222 & 4316 & 2.6892 \\
\hline FIA & 2255 & 2276 & 2.6897 \\
\hline PAC & 1312 & 1219 & 2.6837 \\
\hline IP & 1127 & 1089 & 2.6886 \\
\hline SPR & 758 & 639 & 2.6860 \\
\hline FACS & 608 & 647 & 2.6907 \\
\hline Other & 502 & 495 & 2.6813 \\
\hline SAC & 484 & 393 & 2.6878 \\
\hline ELISPOT & 396 & 412 & 2.6979 \\
\hline RDAT & 366 & 323 & 2.6859 \\
\hline EDAT & 284 & 330 & 2.6800 \\
\hline $\mathrm{XRC}$ & 231 & 227 & 2.6880 \\
\hline MS & 209 & 179 & 2.6849 \\
\hline PFF & 171 & 153 & 2.6820 \\
\hline AbDPO & 162 & 295 & 2.6968 \\
\hline $\mathrm{CdC}$ & 146 & 205 & 2.6895 \\
\hline IAbBA & 144 & 183 & 2.6940 \\
\hline IOT & 124 & 106 & 2.6835 \\
\hline HAGGI & 115 & 122 & 2.6834 \\
\hline IgMHR & 89 & 90 & 2.6929 \\
\hline EAAA & 84 & 139 & 2.6922 \\
\hline HS & 82 & 67 & 2.6791 \\
\hline AbdCC & 73 & 118 & 2.6897 \\
\hline AGG & 50 & 60 & 2.6980 \\
\hline $\mathrm{CM}$ & 50 & 57 & 2.6863 \\
\hline
\end{tabular}

The ${ }^{*}$ indicates that quantities like ${ }^{*} \chi$ is the average value of the mean electronegativity $\left(m_{i}\right)$ for all the peptides in IEDB that are epitopes for the same boundary condition.

\section{Results and Discussion}

We propose herein, for the first time, a QSRR-perturbation model able to predict variations in the propensity of a peptide to act as B-epitope taking into consideration the propensity of a peptide of reference and the changes in peptide sequence, immunological process, host organism, source organisms, and the experimental technique used. The best QSPRperturbation model found here with LDA was

$$
\begin{gathered}
\lambda\left(\varepsilon_{i j}\right)_{\mathrm{new}}=4.979 \cdot \lambda\left(\varepsilon_{i j}\right)_{\mathrm{ref}}-221.642 \cdot \Delta \chi_{\mathrm{seq}} \\
+8.770 \cdot \Delta \Delta \chi_{\mathrm{ho}}+63.572 \cdot \Delta \Delta \chi_{\mathrm{so}} \\
-55.387 \cdot \Delta \Delta \chi_{\mathrm{ip}}+201.919 \cdot \Delta \Delta \chi_{\mathrm{tq}}-2.149 \\
N=155169, \quad R c=0.92 \\
U=0.15, \quad p<0.01 .
\end{gathered}
$$




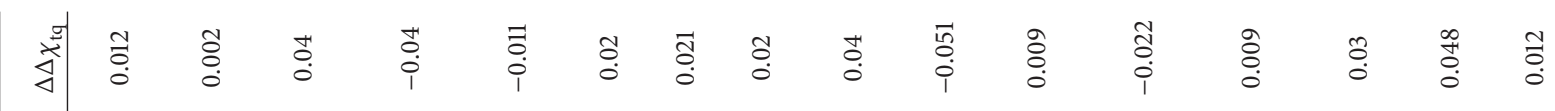

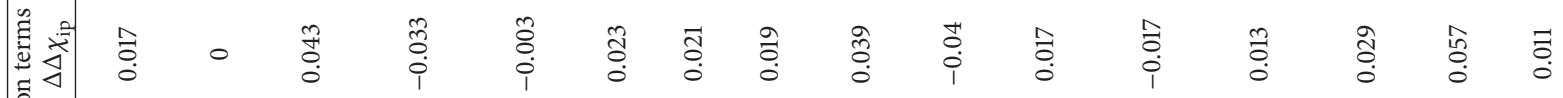

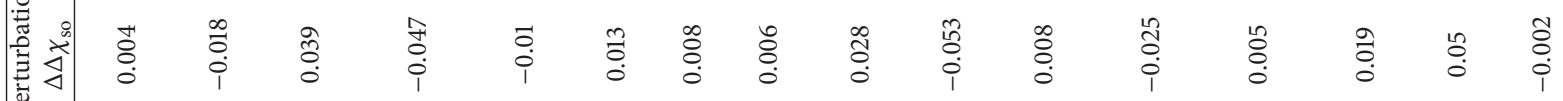

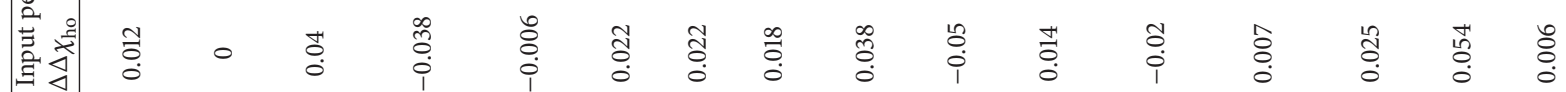

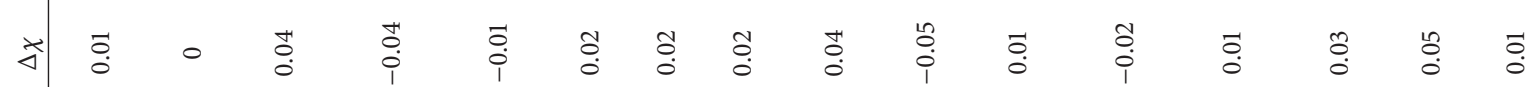

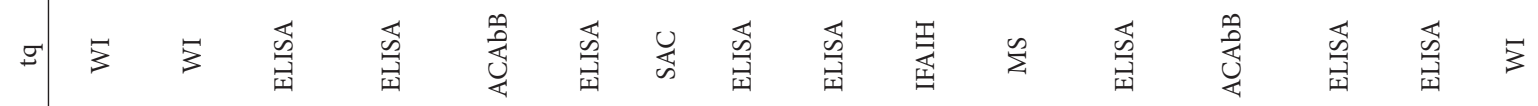

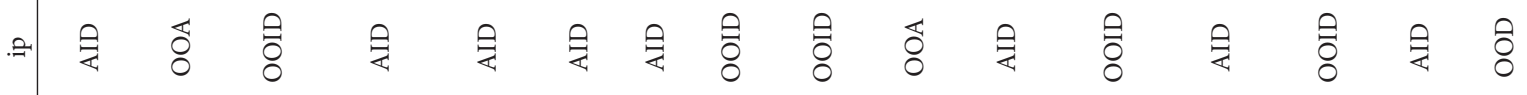

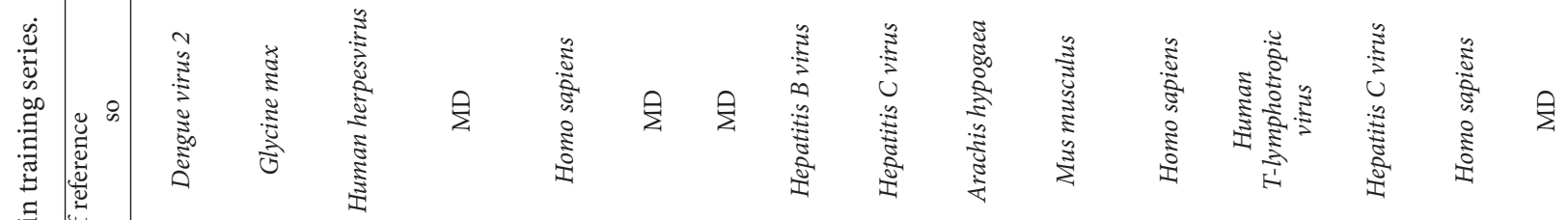

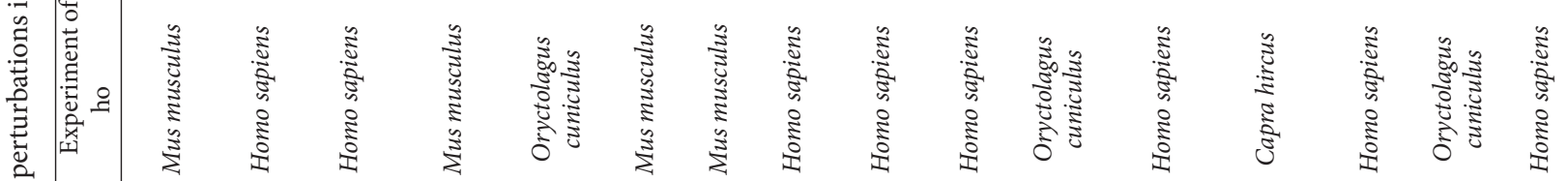

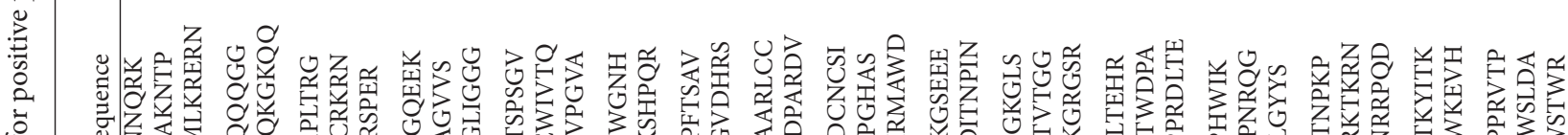

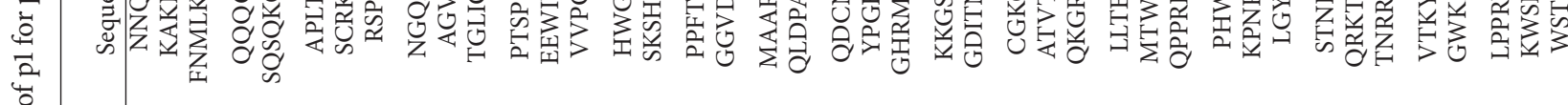

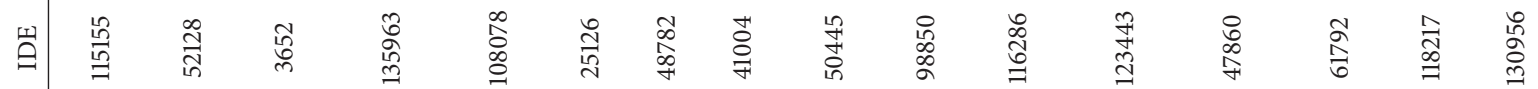

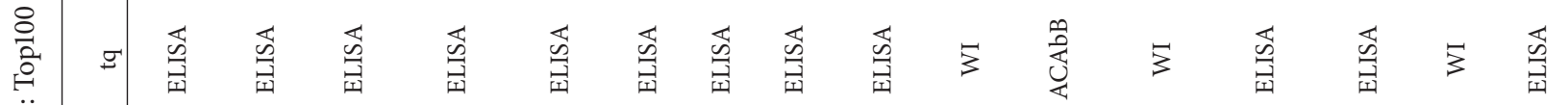

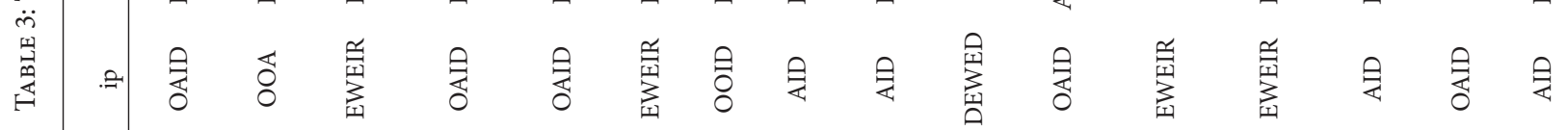

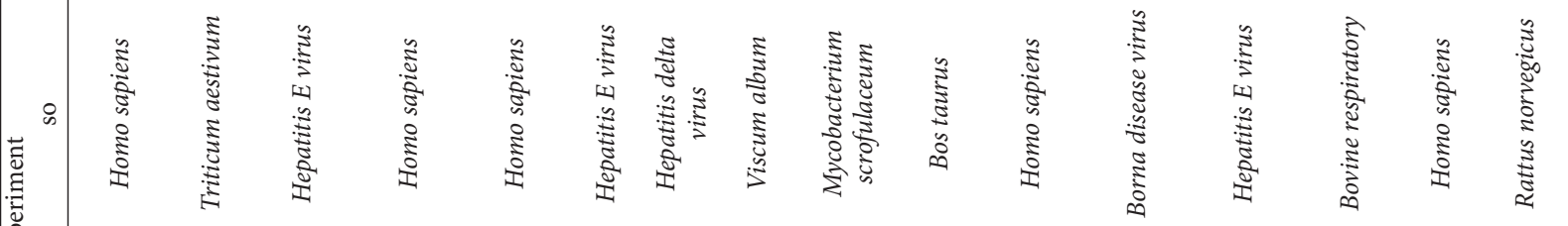

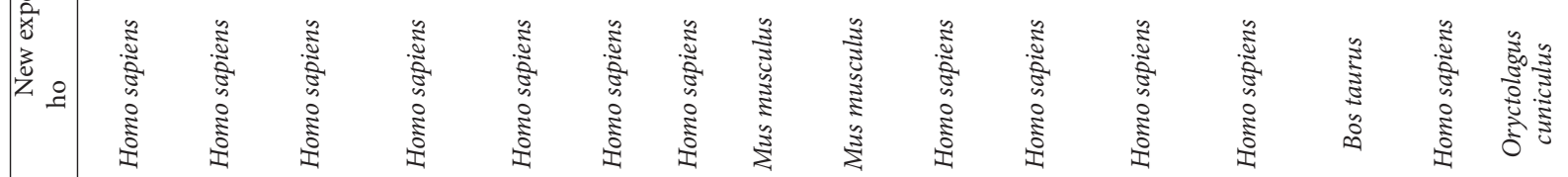

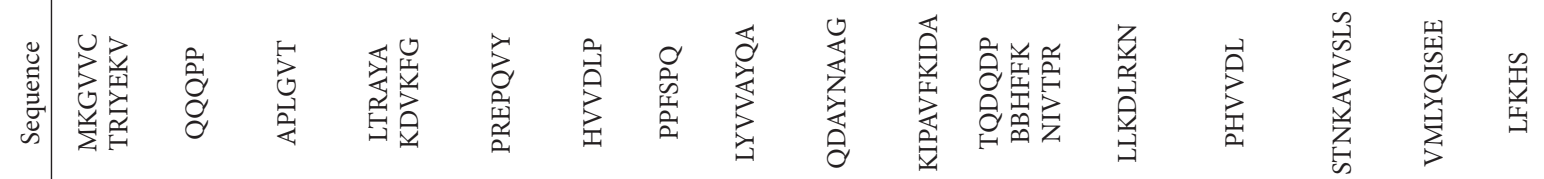

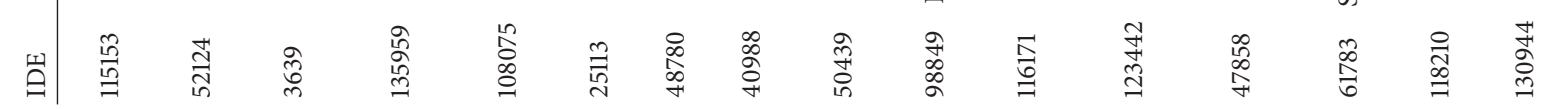




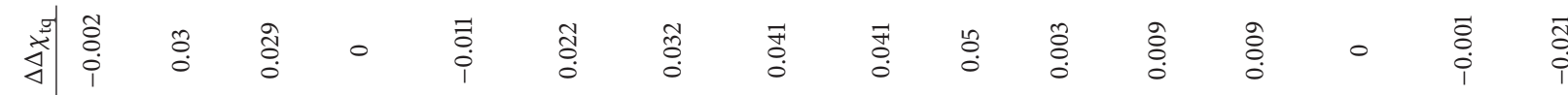

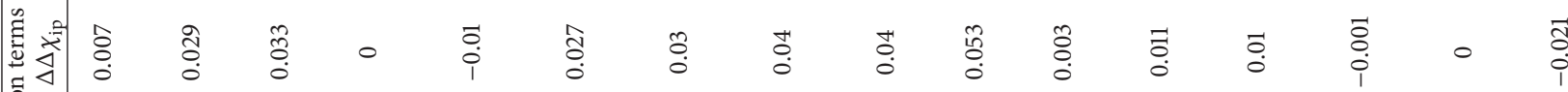

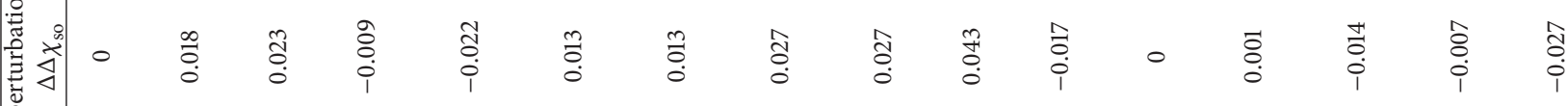

言童。哭

子๐

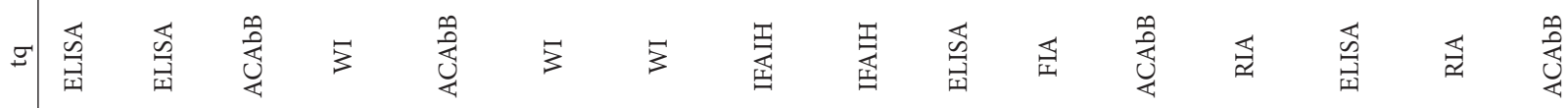

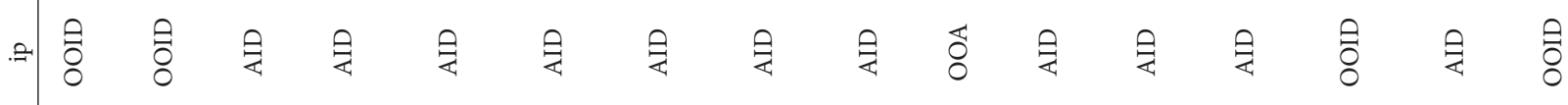

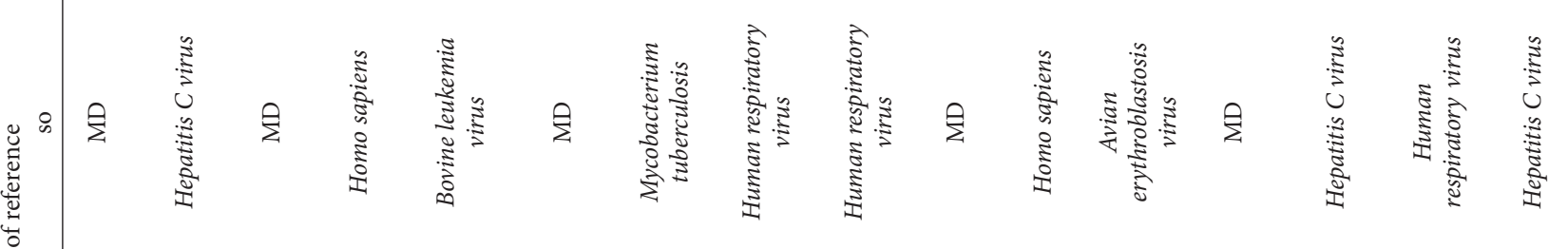

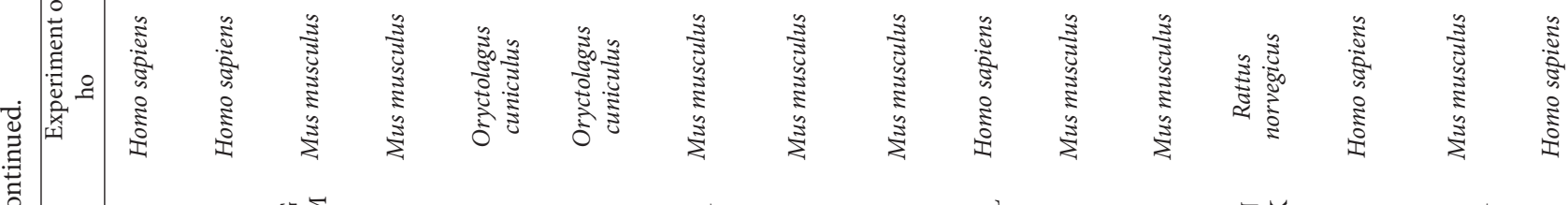

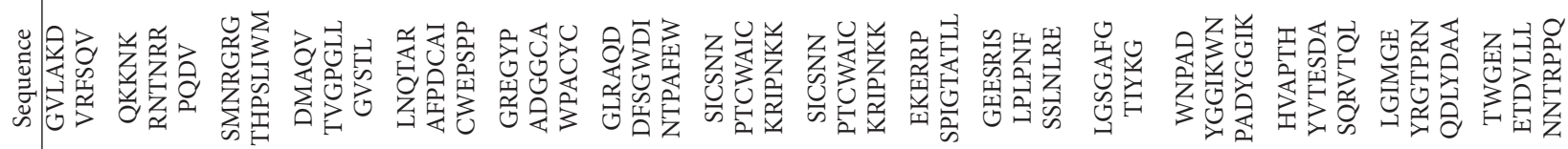

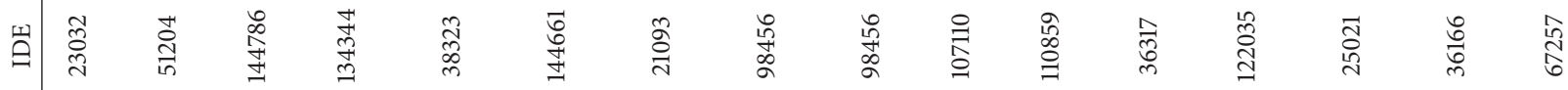

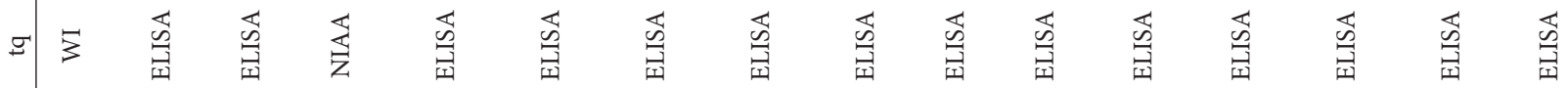

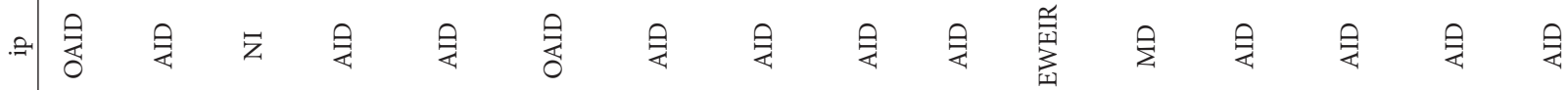

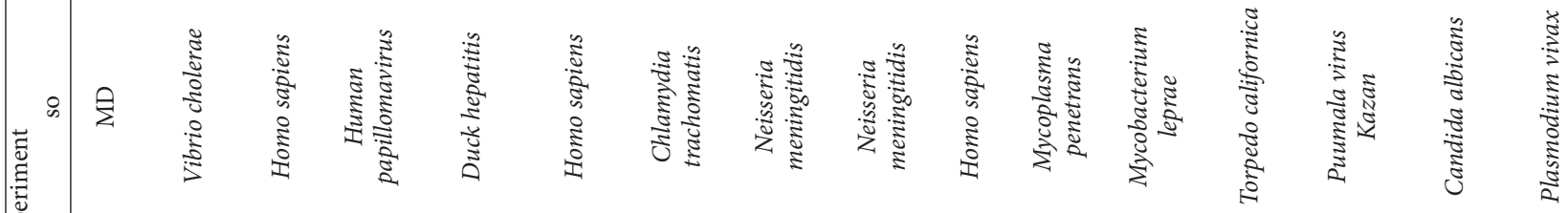

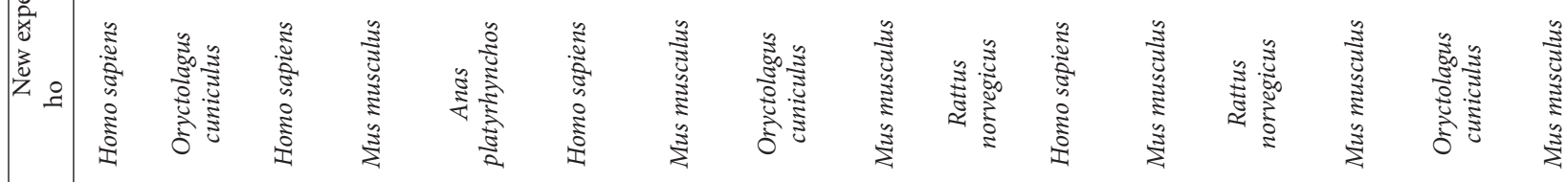

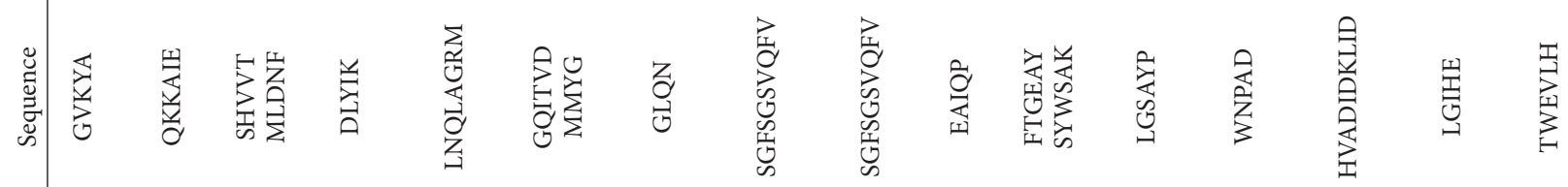

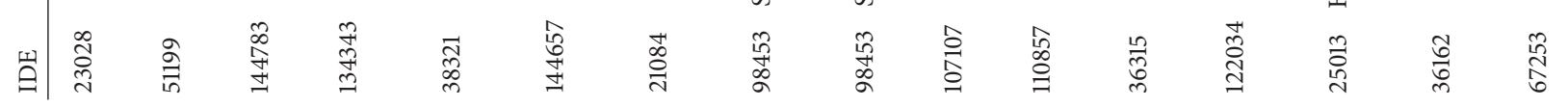




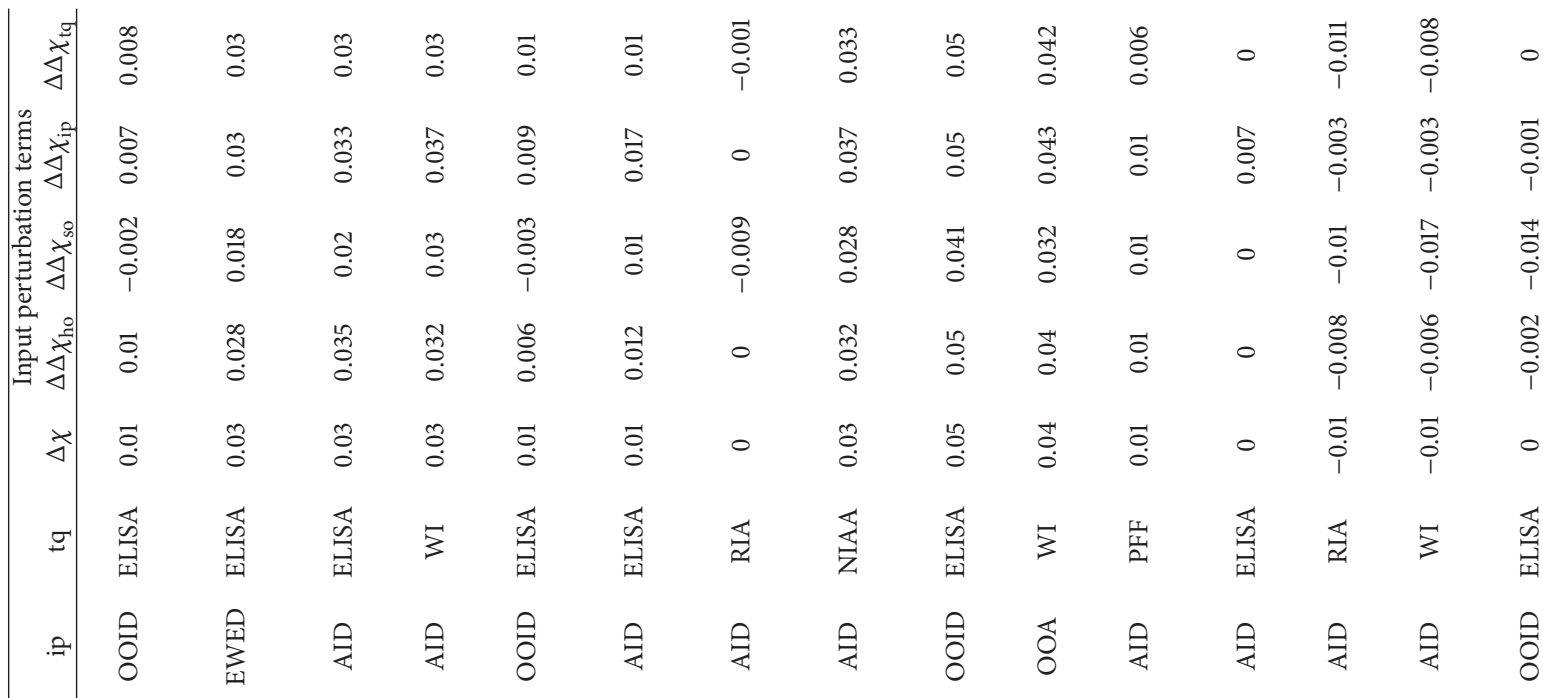

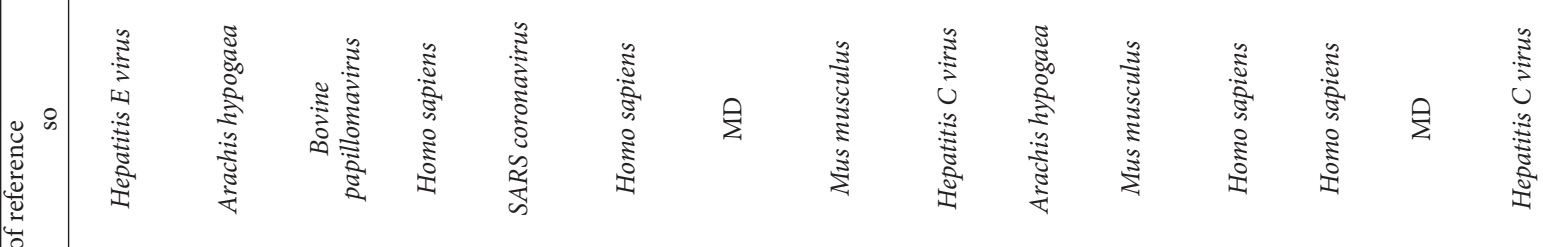

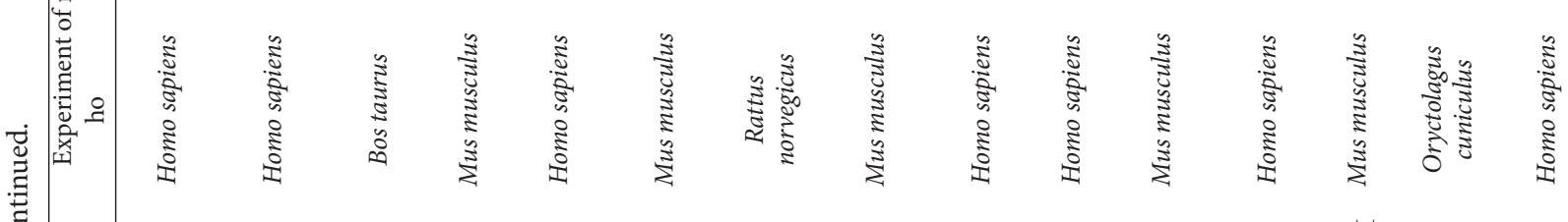

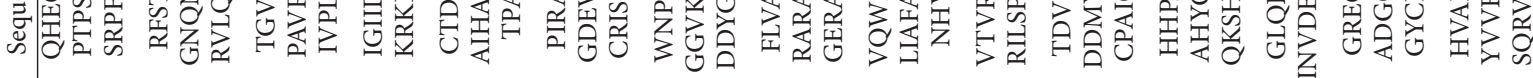

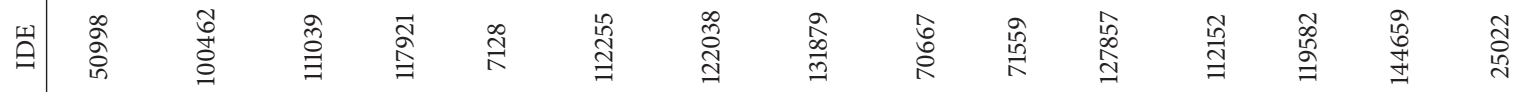

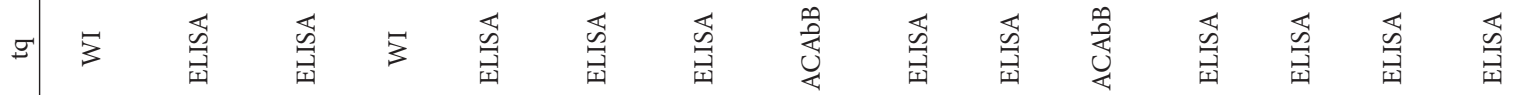

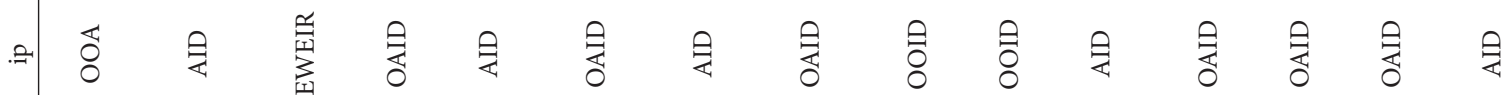

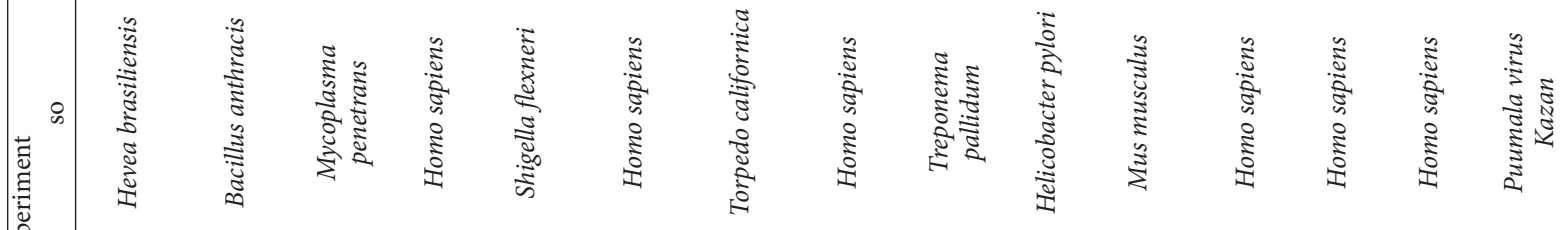

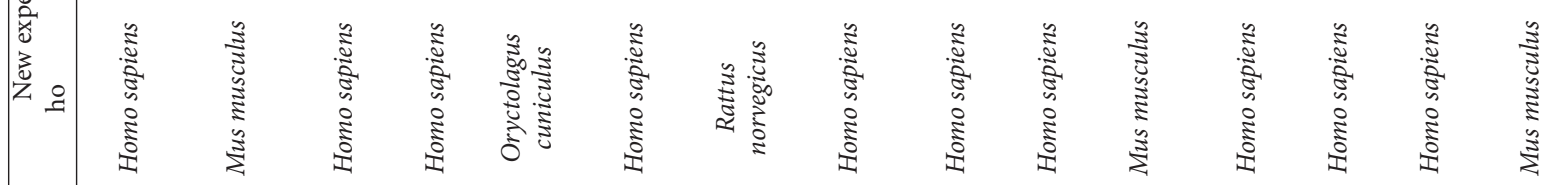

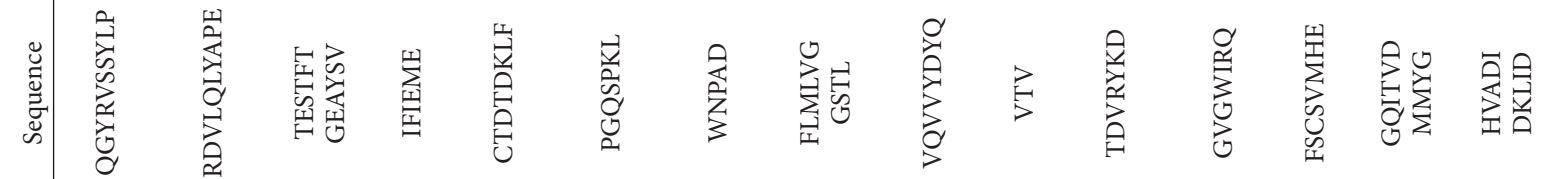

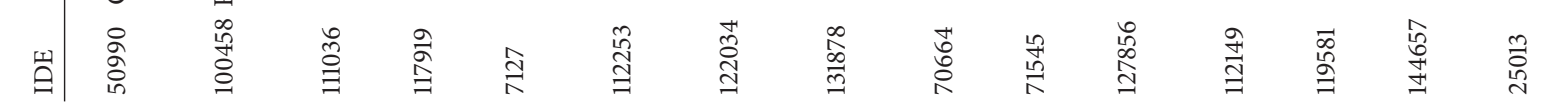




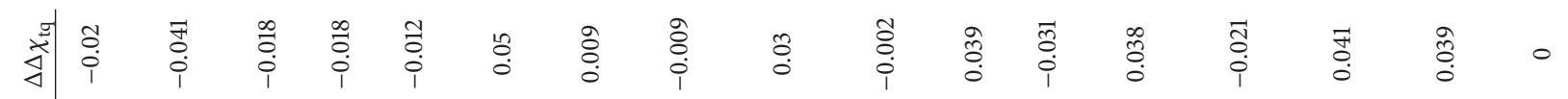

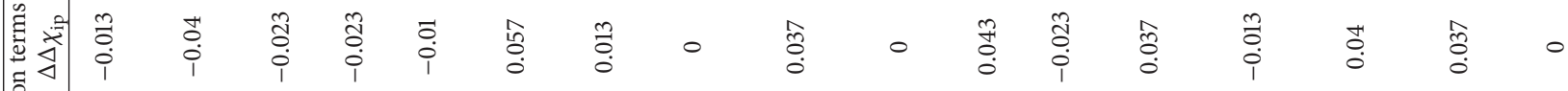

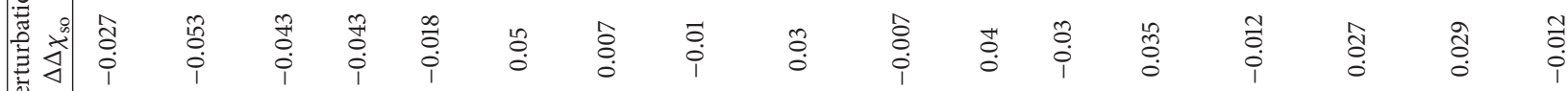

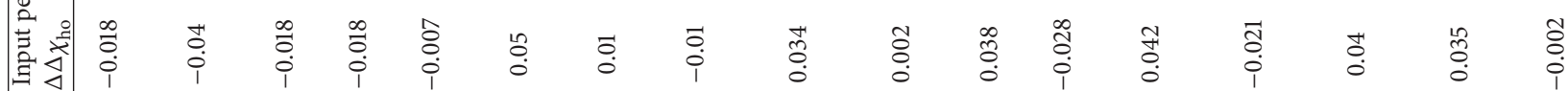

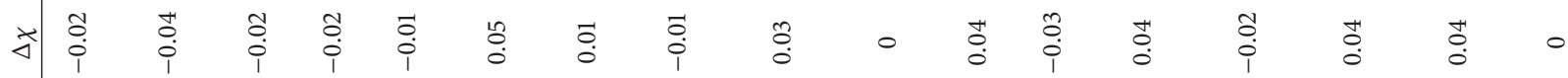

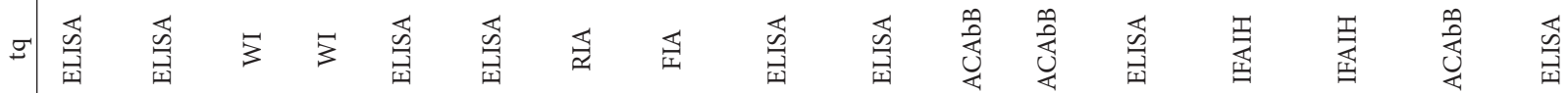

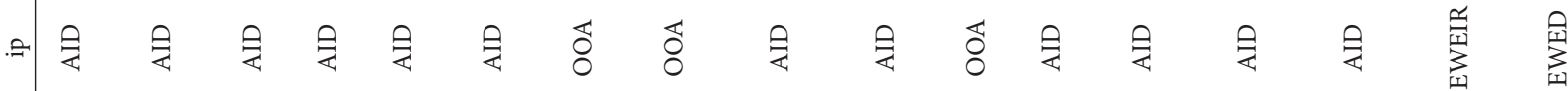

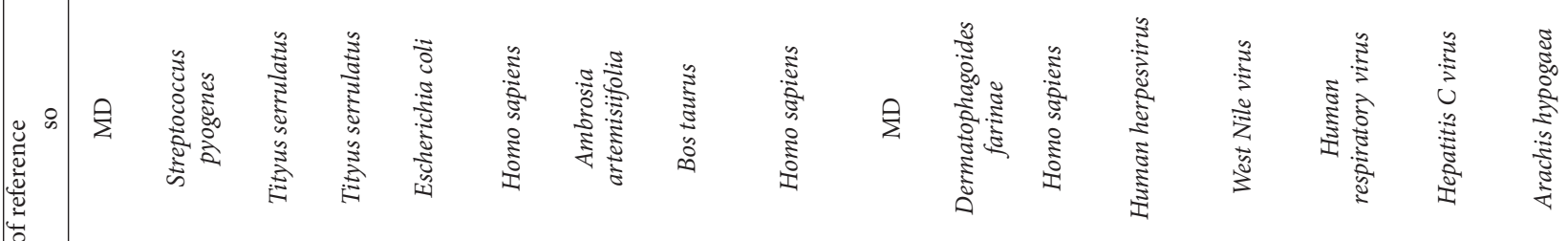

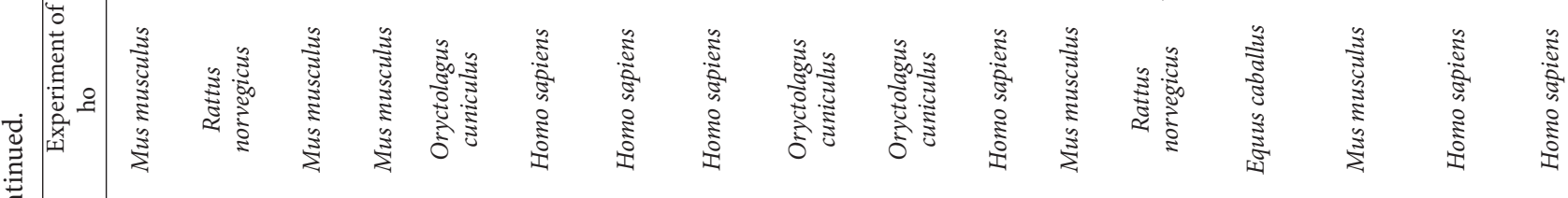

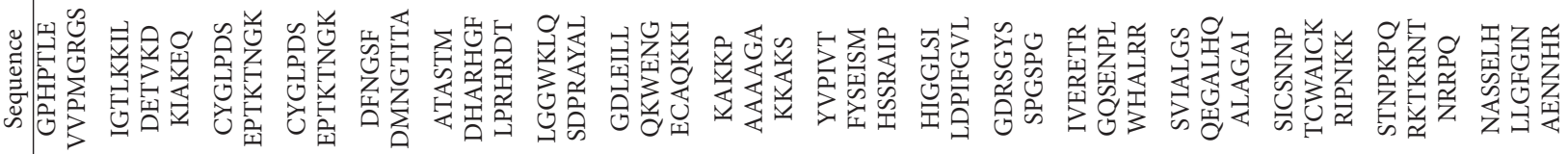

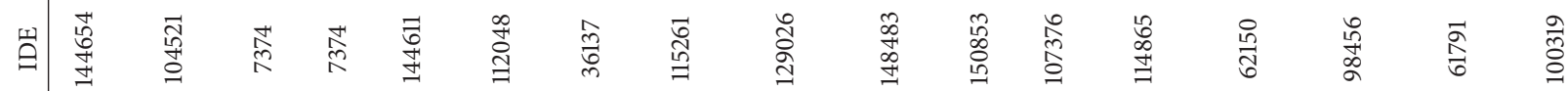

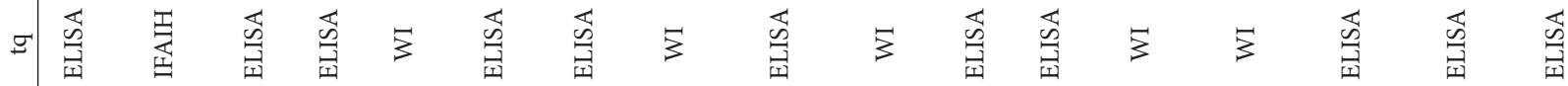

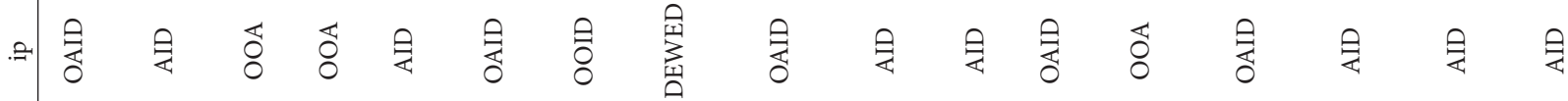

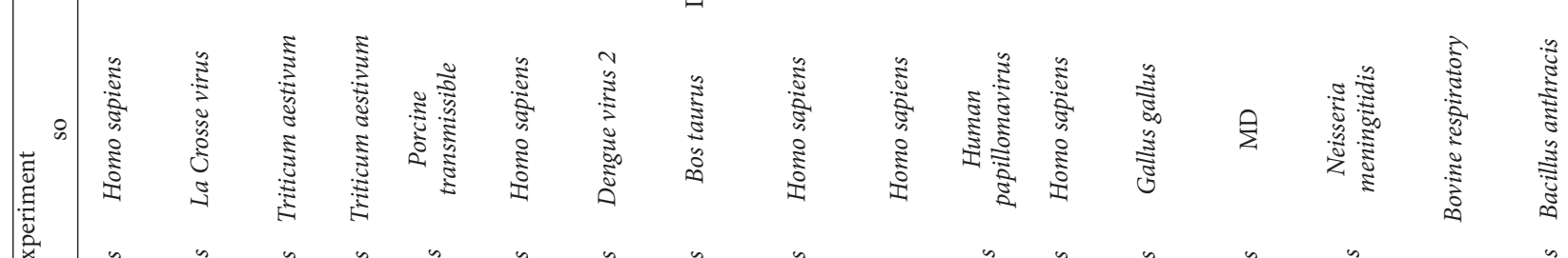

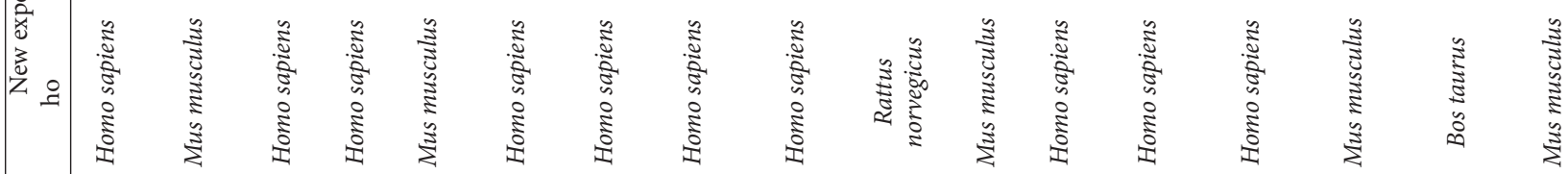

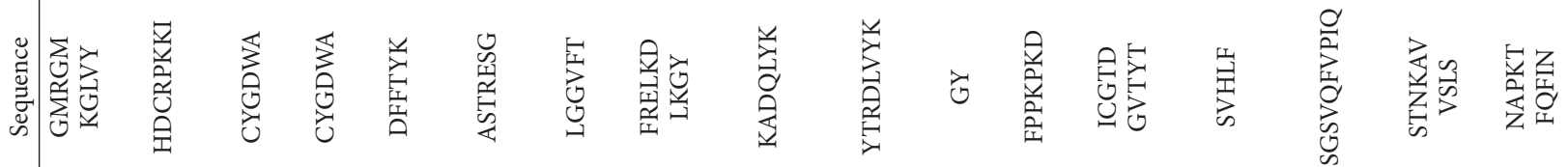

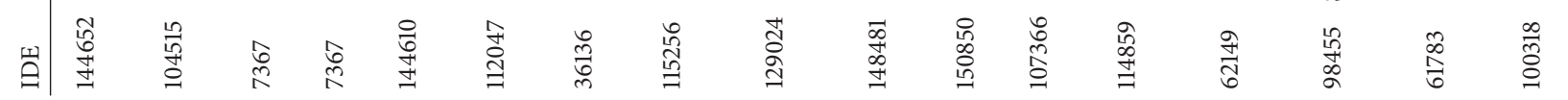




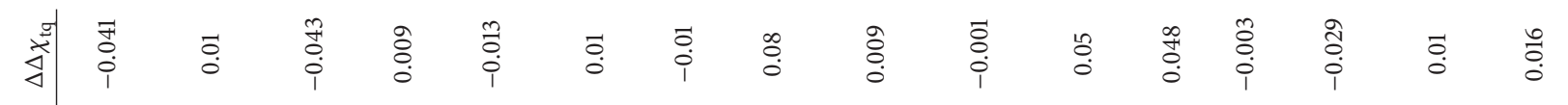

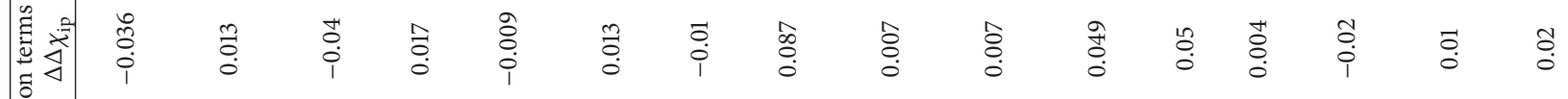

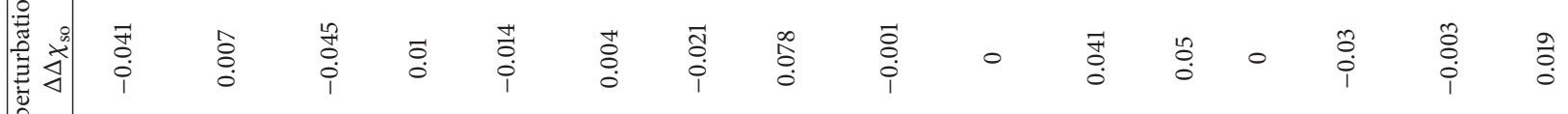

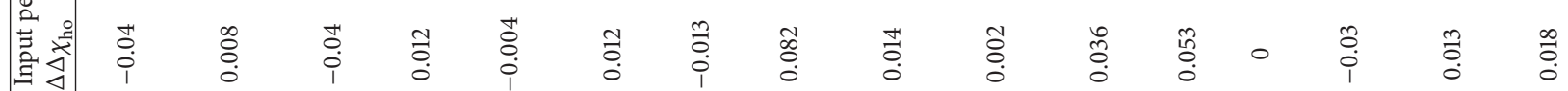

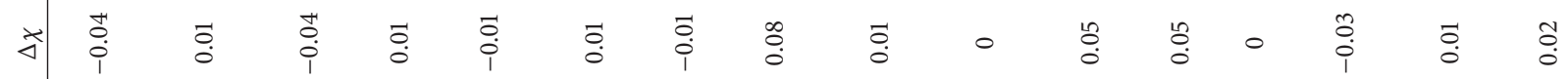

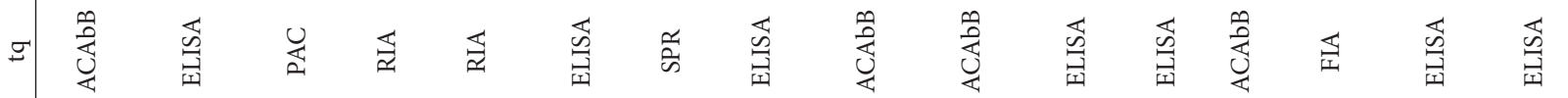

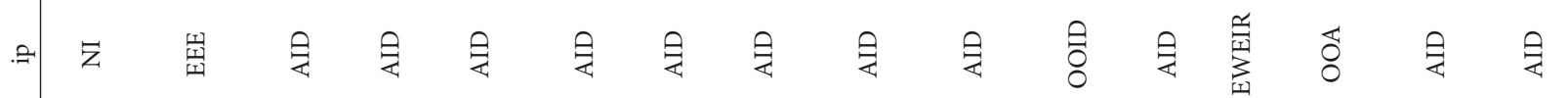

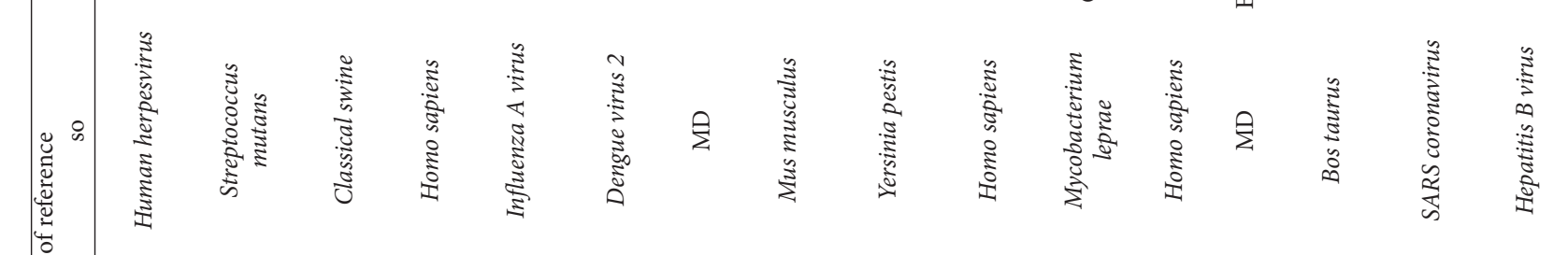

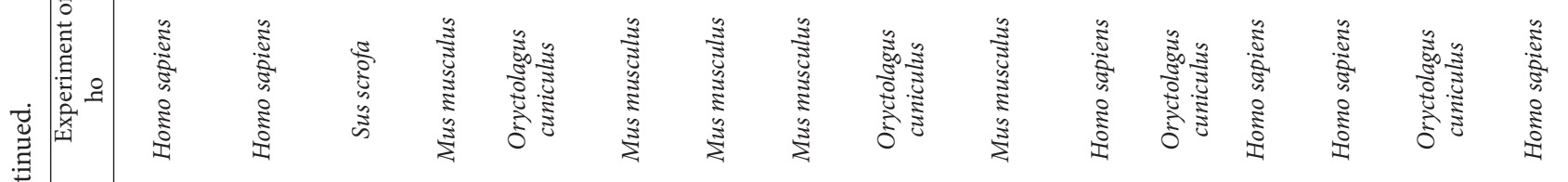

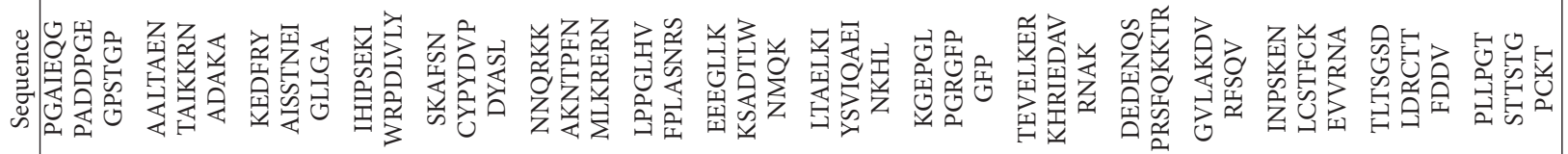

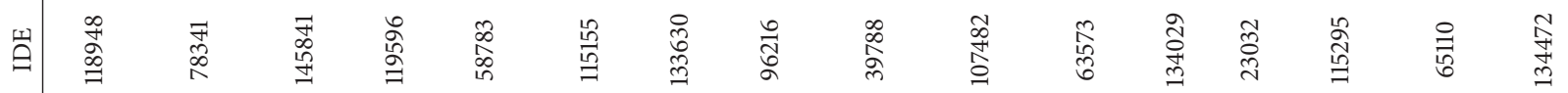

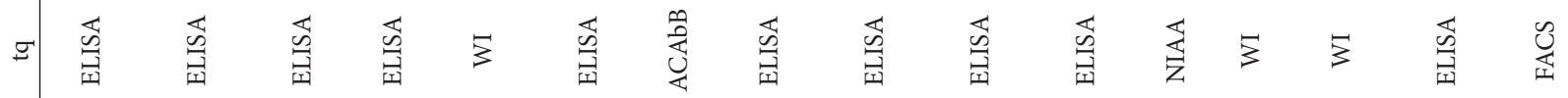

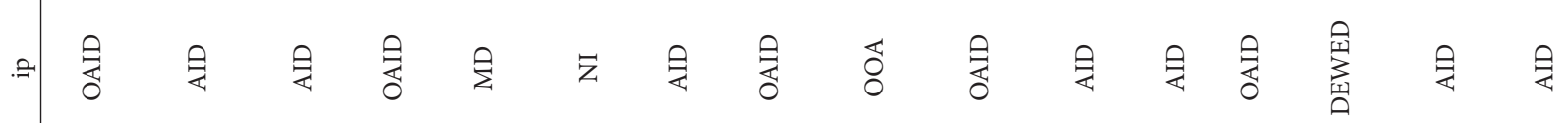

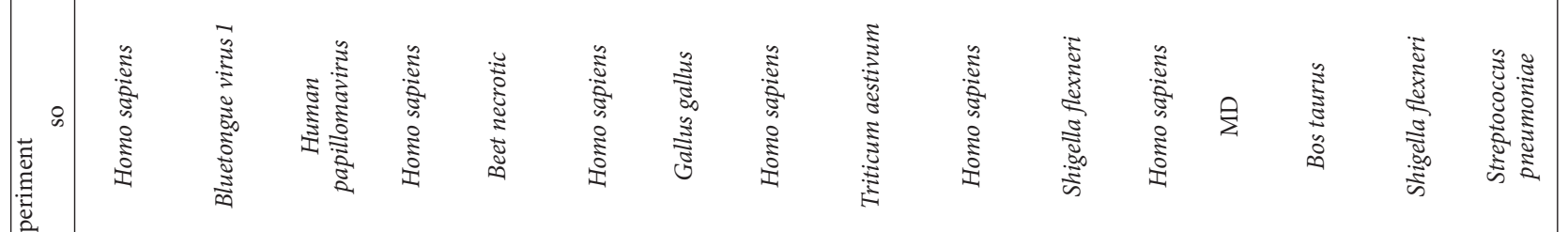

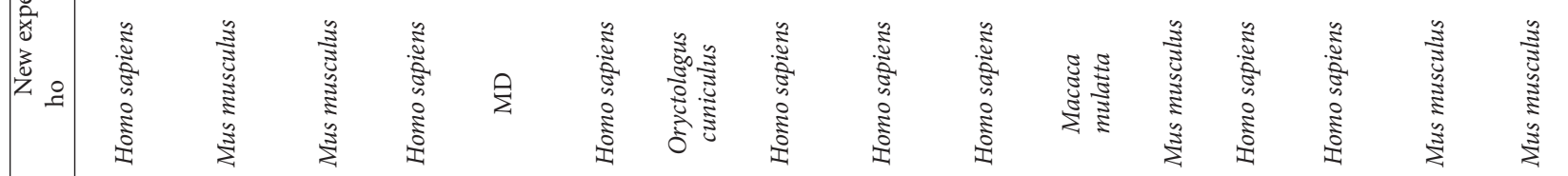

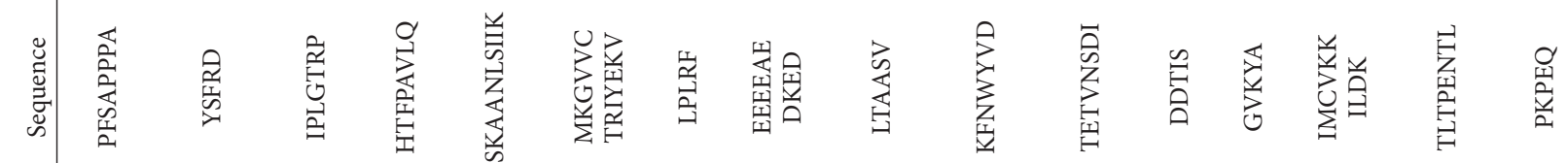

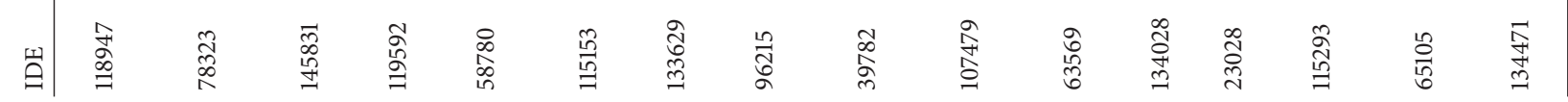




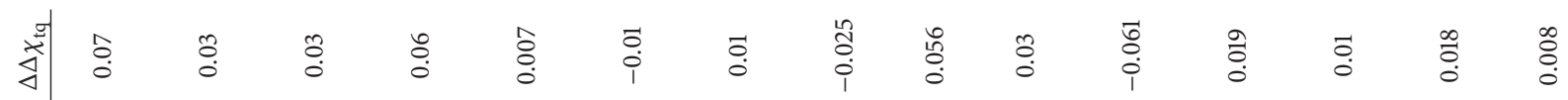

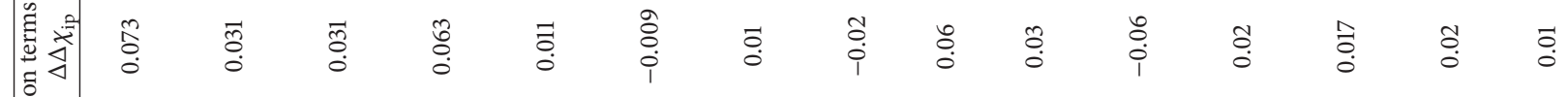

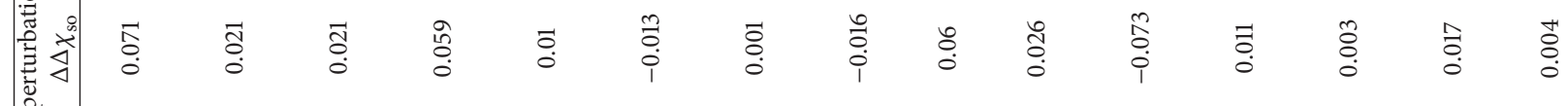

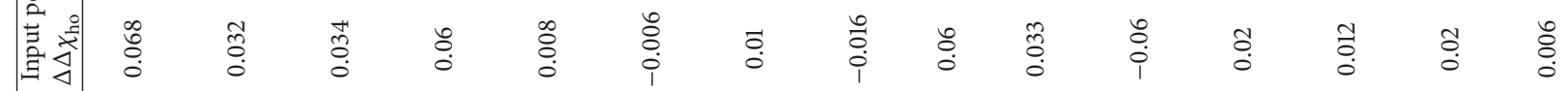

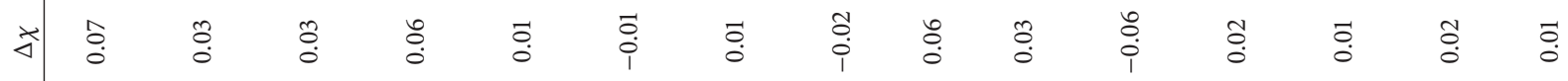

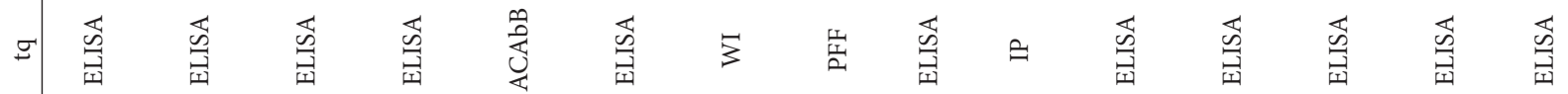

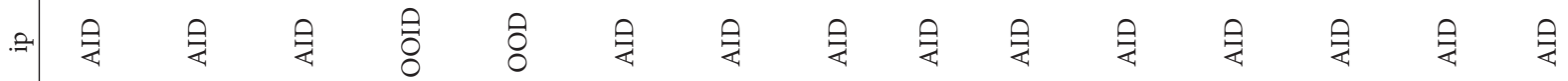

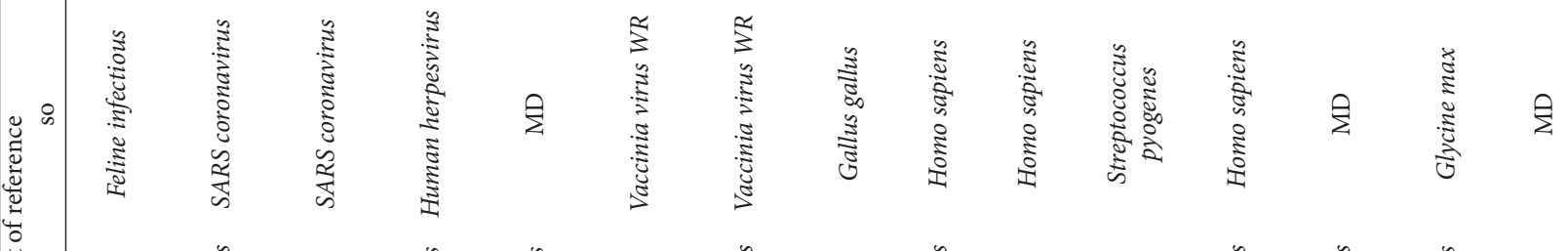

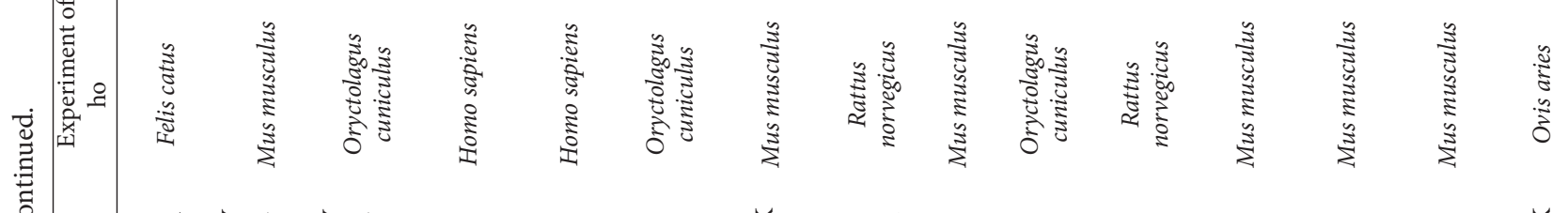

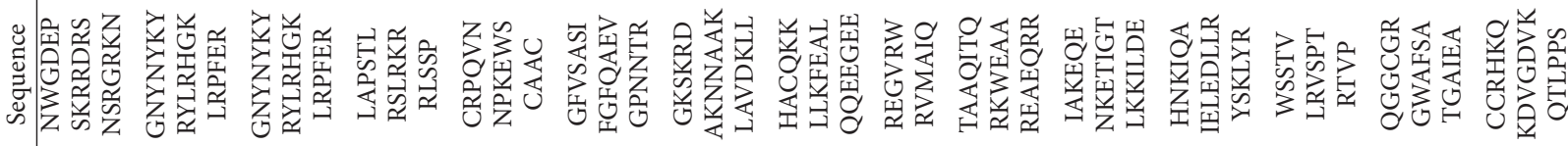

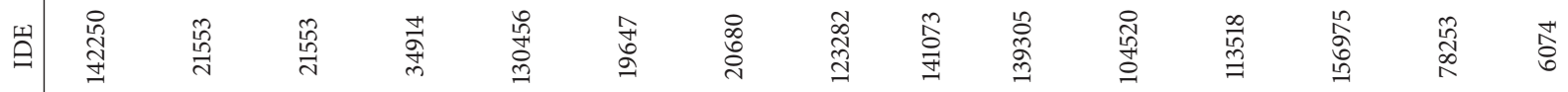

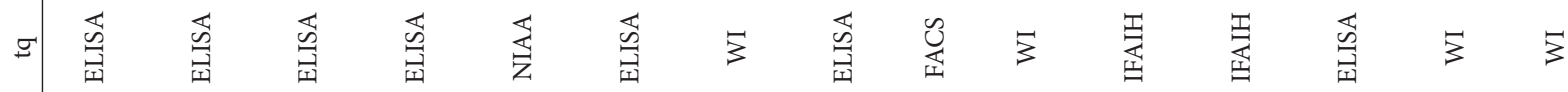

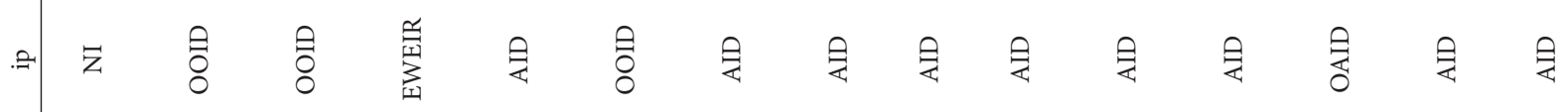

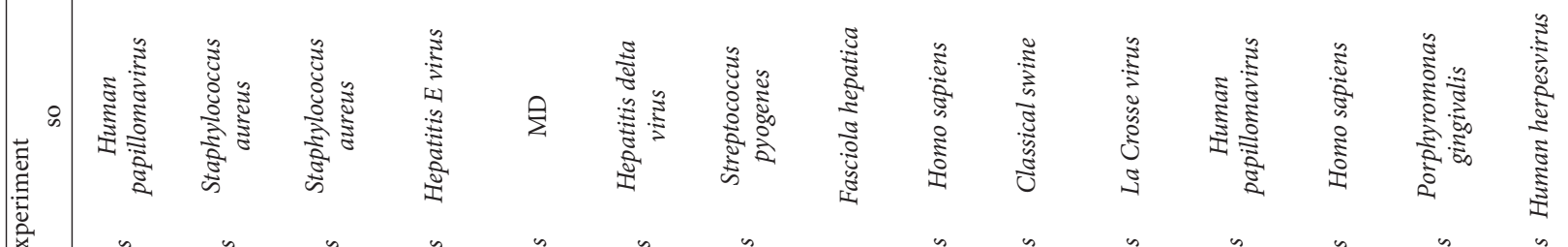
.

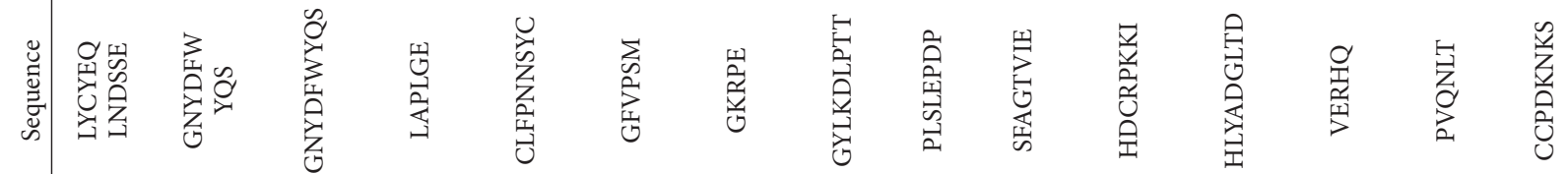

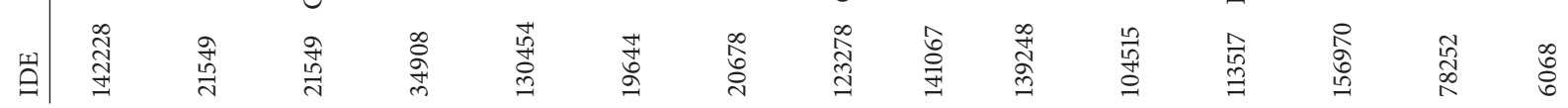




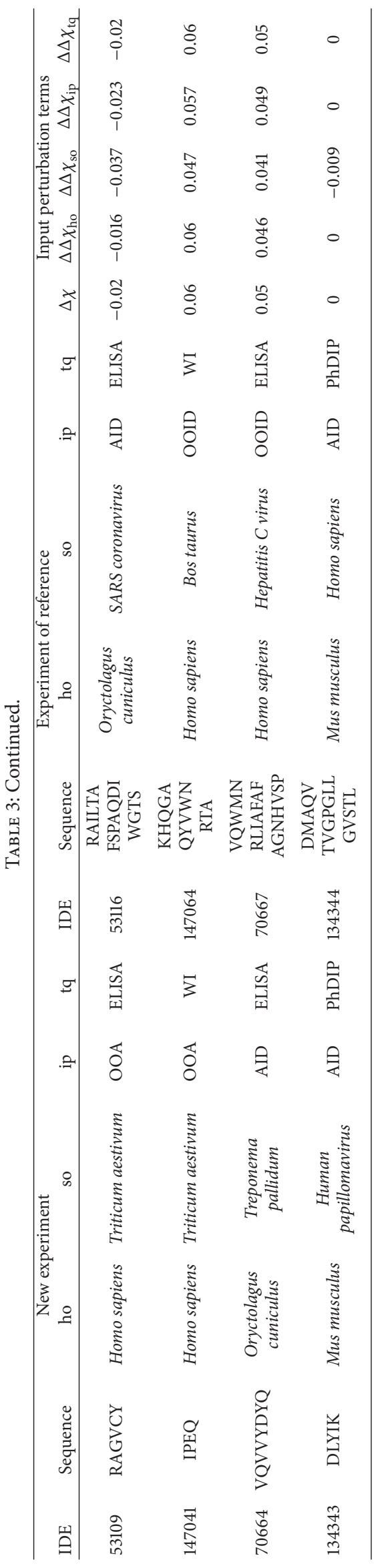


The first input term is the value $\lambda\left(\varepsilon_{i j}\right)_{\text {ref }}$ is the scoring function $\lambda$ of the efficiency of the initial process $\varepsilon_{i j}$ (known solution). The function $\lambda\left(\varepsilon_{i j}\right)_{\text {ref }}=1$ if the $i$ th peptide could experimentally be demonstrated to be a B-epitope in the assay of reference (reference) carried out in the conditions $c_{j}, \lambda\left(\varepsilon_{i j}\right)_{\text {ref }}=0$ otherwise. The variational-perturbation terms $\Delta \Delta \chi_{c j}$ are at the same time terms typical of perturbation theory and moving average (MA) functions used in Box-Jenkin models in time series [60]. These new types of terms account both for the deviation of the electronegativity of all amino acids in the sequence of the new peptide with respect to the peptide of reference and with respect to all boundary conditions. In Table 1, we give the overall classification results obtained with this model. Speck-Planche et al. [61-63] introduced different multitarget/multiplexing QSAR models that incorporate this type of information based on MAs. The results obtained with the present model are excellent compared with other similar models in the literature useful for other problems including moving average models $[64,65]$ or perturbation models [58]. Notably, this is also the first model combining both perturbation theory and MAs in a QSPR context.

The other input terms are the following. The first $\Delta \chi_{\text {seq }}=$ $\chi\left(m_{q}\right)_{\text {ref }}-\chi\left(m_{i}\right)_{\text {new }}$ is the perturbation term for the variation or in the mean value of electronegativity for all amino acids in the sequence of the peptide of reference. The remnant input variables of the model $\Delta \Delta \chi_{c j}=\Delta \chi_{c j \text {-ref }}-\Delta \chi_{c j \text {-new }}=$ $\left[\chi\left(m_{q}\right)_{\text {ref }}-{ }^{*} \chi\left(c_{q r}\right)_{\text {ref }}\right]-\left[\chi\left(m_{i}\right)_{\text {new }}-{ }^{*} \chi\left(c_{i j}\right)_{\text {new }}\right]$ quantify values of the conditions of the new assay $c j$-new that represent perturbations with respect to the initial conditions $c_{i j}$-ref of the assay of reference. The quantities ${ }^{*} \chi\left(c_{i j}\right)$ and ${ }^{*} \chi\left(c_{q r}\right)$ are the average values of the mean electronegativity values $\chi\left(m_{i}\right)$ and $\chi\left(m_{q}\right)$ for all new and reference peptides in IEDB that are epitopes under the $j$ th or $r$ th boundary condition. The values of these terms have been tabulated for $>500$ source organisms, $>50$ host organisms, $>10$ biological process, and $>30$ experimental techniques. We must substitute the values of $\chi\left(m_{i}\right)$ and $\chi\left(m_{q}\right)$ of the new and reference peptides and the tabulated values of ${ }^{*} \chi\left(c_{i j}\right)$ and ${ }^{*} \chi\left(c_{q r}\right)$ for all combinations of boundary conditions to predict the perturbations of the action as epitope of peptides. In doing so we can found the optimal sequence and boundary conditions towards the use of the peptide in the development of a vaccine. In Table 2 we give some of these values of ${ }^{*} \chi\left(c_{i j}\right)$ and ${ }^{*} \chi\left(c_{q r}\right)$.

In Table 3 we depict the sequences and input-output boundary conditions for top perturbations present in IEDB. All these perturbations have observed value of $\lambda\left(\varepsilon_{i j}\right)_{\text {new }}=1$ and predicted value also equal to 1 with a high probability. See Supplementary Material available online at http://dx.doi .org/10.1155/2014/768515 file contains a full list of $>200,000$ cases of perturbations.

\section{Conclusions}

It is possible to develop general models for vaccine design able to predict the results of multiple input-output perturbations in peptide sequence and experimental assay boundary conditions using ideas of QSPR analysis, perturbation theory, and Box and Jenkins MA operators. The electronegativity values calculated with MARCH-INSIDE seem to be good molecular descriptors for this type of QSPR-perturbation models.

\section{Conflict of Interests}

The authors declare that there is no conflict of interests regarding the publication of this paper.

\section{Acknowledgments}

The present study was partially supported by Grants AGL2010-22290-C02 and AGL2011-30563-C03 from Ministerio de Ciencia e Innovación, Spain, and Grant CN 2012/155 from Xunta de Galicia, Spain.

\section{References}

[1] B. Peters, J. Sidney, P. Bourne et al., "The design and implementation of the immune epitope database and analysis resource," Immunogenetics, vol. 57, no. 5, pp. 326-336, 2005.

[2] B. Peters, J. Sidney, P. Bourne et al., "The immune epitope database and analysis resource: from vision to blueprint," PLoS Biology, vol. 3, no. 3, p. e91, 2005.

[3] P. Wang, A. A. Morgan, Q. Zhang, A. Sette, and B. Peters, "Automating document classification for the Immune Epitope Database," BMC Bioinformatics, vol. 8, article 269, 2007.

[4] A. Sette, "The immune epitope database and analysis resource: from vision to blueprint," Genome Informatics, vol. 15, no. 2, p. 299, 2004.

[5] N. Salimi, W. Fleri, B. Peters, and A. Sette, "The immune epitope database: a historical retrospective of the first decade," Immunology, vol. 137, no. 2, pp. 117-123, 2012.

[6] Y. Kim, A. Sette, and B. Peters, "Applications for T-cell epitope queries and tools in the Immune Epitope Database and Analysis Resource," Journal of Immunological Methods, vol. 374, no. 1-2, pp. 62-69, 2011.

[7] Y. Kim, J. Ponomarenko, Z. Zhu, D. Tamang et al., "Immune epitope database analysis resource," Nucleic Acids Research, pp. W525-W530, 2012.

[8] A. M. Helguera, R. D. Combes, M. P. Gonzalez, and M. N. D. S. Cordeiro, "Applications of 2D descriptors in drug design: a DRAGON tale," Current Topics in Medicinal Chemistry, vol. 8, no. 18, pp. 1628-1655, 2008.

[9] G. M. Casañola-Martín, Y. Marrero-Ponce, M. T. H. Khan et al., "Dragon method for finding novel tyrosinase inhibitors: Biosilico identification and experimental in vitro assays," The European Journal of Medicinal Chemistry, vol. 42, no. 11-12, pp. 13701381, 2007.

[10] I. V. Tetko, J. Gasteiger, R. Todeschini et al., "Virtual computational chemistry laboratory-design and description," Journal of Computer-Aided Molecular Design, vol. 19, no. 6, pp. 453-463, 2005.

[11] A. R. Katritzky, A. Oliferenko, A. Lomaka, and M. Karelson, "Six-membered cyclic ureas as HIV-1 protease inhibitors: a QSAR study based on CODESSA PRO approach," Bioorganic and Medicinal Chemistry Letters, vol. 12, no. 23, pp. 3453-3457, 2002. 
[12] A. R. Katritzky, S. Perumal, R. Petrukhin, and E. Kleinpeter, "CODESSA-based theoretical QSPR model for hydantoin HPLC-RT lipophilicities," Journal of Chemical Information and Computer Sciences, vol. 41, no. 3, pp. 569-574, 2001.

[13] S. Vilar, G. Cozza, and S. Moro, "Medicinal chemistry and the molecular operating environment (MOE): application of QSAR and molecular docking to drug discovery," Current Topics in Medicinal Chemistry, vol. 8, no. 18, pp. 1555-1572, 2008.

[14] G. Marzaro, A. Chilin, A. Guiotto et al., "Using the TOPSMODE approach to fit multi-target QSAR models for tyrosine kinases inhibitors," The European Journal of Medicinal Chemistry, vol. 46, no. 6, pp. 2185-2192, 2011.

[15] S. Vilar, E. Estrada, E. Uriarte, L. Santana, and Y. Gutierrez, "In silico studies toward the discovery of new anti-HIV nucleoside compounds through the use of tops-mode and 2D/3D connectivity indices. 2. Purine derivatives," Journal of Chemical Information and Modeling, vol. 45, no. 2, pp. 502-514, 2005.

[16] E. Estrada, J. A. Quincoces, and G. Patlewicz, "Creating molecular diversity from antioxidants in Brazilian propolis. combination of TOPS-MODE QSAR and virtual structure generation," Molecular Diversity, vol. 8, no. 1, pp. 21-33, 2004.

[17] E. Estrada and H. González, "What are the limits of applicability for graph theoretic descriptors in QSPR/QSAR? modeling dipole moments of aromatic compounds with TOPS-MODE descriptors," Journal of Chemical Information and Computer Sciences, vol. 43, no. 1, pp. 75-84, 2003.

[18] Y. Marrero-Ponce, J. A. Castillo-Garit, E. Olazabal et al., "Tomocomd-Cardd, a novel approach for computer-aided "rational" drug design: I. Theoretical and experimental assessment of a promising method for computational screening and in silico design of new anthelmintic compounds," Journal of ComputerAided Molecular Design, vol. 18, no. 10, pp. 615-634, 2004.

[19] Y. Marrero Ponce, R. Medina Marrero, E. A. Castro et al., "Protein quadratic indices of the "macromolecular pseudograph's $\alpha$ carbon atom adjacency matrix". 1. prediction of arc repressor alanine-mutant's stability," Molecules, vol. 9, no. 12, pp.1124-1147, 2004.

[20] H. González-Díaz, F. Prado-Prado, and F. M. Ubeira, "Predicting antimicrobial drugs and targets with the MARCH-INSIDE approach," Current Topics in Medicinal Chemistry, vol. 8, no. 18, pp. 1676-1690, 2008.

[21] R. García-Domenech, J. Gálvez, J. V. de Julián-Ortiz, and L. Pogliani, "Some new trends in chemical graph theory," Chemical Reviews, vol. 108, no. 3, pp. 1127-1169, 2008.

[22] E. Estrada and E. Uriarte, "Recent advances on the role of topological indices in drug discovery research," Current Medicinal Chemistry, vol. 8, no. 13, pp. 1573-1588, 2001.

[23] R. Todeschini and V. Consonni, Handbook of Molecular Descriptors, Wiley-VCH, Weinheim, Germany, 2008.

[24] E. Estrada, E. J. Delgado, J. B. Alderete, and G. A. Jaña, "Quantum-connectivity descriptors in modeling solubility of environmentally important organic compounds," Journal of Computational Chemistry, vol. 25, no. 14, pp. 1787-1796, 2004.

[25] E. Besalu, X. Girones, L. Amat, and R. Carbo-Dorca, "Molecular quantum similarity and the fundamentals of QSAR," Accounts of Chemical Research, vol. 35, no. 5, pp. 289-295, 2002.

[26] D. A. Rincón, M. N. D. S. Cordeiro, and R. A. Mosquera, "On the electronic structure of cocaine and its metabolites," Journal of Physical Chemistry A, vol. 113, no. 50, pp. 13937-13942, 2009.

[27] M. Mandado, M. J. González-Moa, and R. A. Mosquera, "Chemical graph theory and n-center electron delocalization indices: a study on polycyclic aromatic hydrocarbons," Journal of Computational Chemistry, vol. 28, no. 10, pp. 1625-1633, 2007.

[28] T. Hill and P. Lewicki, STATISTICS: Methods and Applications: A Comprehensive Reference for Science, Industry and Data Mining, StatSoft, Tulsa, Okla, USA, 2006.

[29] E. Frank, M. Hall, L. Trigg, G. Holmes, and I. H. Witten, "Data mining in bioinformatics using Weka," Bioinformatics, vol. 20, no. 15, pp. 2479-2481, 2004.

[30] G. Patlewicz, N. Ball, E. D. Booth, E. Hulzebos, E. Zvinavashe, and C. Hennes, "Use of category approaches, read-across and (Q)SAR: general considerations," Regulatory Toxicology and Pharmacology, vol. 67, no. 1, pp. 1-12, 2013.

[31] D. W. Roberts and G. Y. Patlewicz, "Updating the skin sensitization in vitro data assessment paradigm in 2009-a chemistry and QSAR perspective," Journal of Applied Toxicology, vol. 30, no. 3, pp. 286-288, 2010.

[32] D. W. Roberts and G. Y. Patlewicz, "Nonanimal alternatives for skin sensitization: letter to the editor," Toxicological Sciences, vol. 106, no. 2, pp. 572-574, 2008.

[33] E. Estrada, G. Patlewicz, and Y. Gutierrez, "From knowledge generation to knowledge archive. a general strategy using TOPS-MODE with DEREK to formulate new alerts for skin sensitization," Journal of Chemical Information and Computer Sciences, vol. 44, no. 2, pp. 688-698, 2004.

[34] G. F. Gerberick, C. A. Ryan, P. S. Kern et al., "A chemical dataset for evaluation of alternative approaches to skin-sensitization testing," Contact Dermatitis, vol. 50, no. 5, pp. 274-288, 2004.

[35] G. Y. Patlewicz, D. A. Basketter, C. K. Smith Pease et al., "Further evaluation of quantitative structure-activity relationship models for the prediction of the skin sensitization potency of selected fragrance allergens," Contact Dermatitis, vol. 50, no. 2, pp. 91-97, 2004.

[36] E. Estrada, G. Patlewicz, M. Chamberlain, D. Basketter, and S. Larbey, "Computer-aided knowledge generation for understanding skin sensitization mechanisms: the TOPS-MODE approach," Chemical Research in Toxicology, vol. 16, no. 10, pp. 1226-1235, 2003.

[37] G. Patlewicz, R. Rodford, and J. D. Walker, "Quantitative structure-activity relationships for predicting skin and eye irritation," Environmental Toxicology and Chemistry, vol. 22, no. 8, pp. 1862-1869, 2003.

[38] G. Y. Patlewicz, R. A. Rodford, G. Ellis, and M. D. Barratt, "A QSAR model for the eye irritation of cationic surfactants," Toxicology In Vitro, vol. 14, no. 1, pp. 79-84, 2000.

[39] E. Tenorio-Borroto, C. G. Penuelas Rivas, J. C. Vasquez Chagoyan, N. Castanedo, F. J. Prado-Prado, and X. GarciaMera, "ANN multiplexing model of drugs effect on macrophages; theoretical and flow cytometry study on the cytotoxicity of the anti-microbial drug G1 in spleen," Bioorganic \& Medicinal Chemistry, vol. 20, no. 20, pp. 6181-6194, 2012.

[40] D. R. Flower, H. McSparron, M. J. Blythe et al., "Computational vaccinology: quantitative approaches," Novartis Foundation Symposium, vol. 254, pp. 102-120, 2003.

[41] I. A. Doytchinova, P. Guan, and D. R. Flower, "Quantitative structure-activity relationships and the prediction of MHC supermotifs," Methods, vol. 34, no. 4, pp. 444-453, 2004.

[42] Y. Xiao and M. R. Segal, "Prediction of genomewide conserved epitope profiles of HIV-1: classifier choice and peptide representation," Statistical Applications in Genetics and Molecular Biology, vol. 4, no. 1, article 25, 2005. 
[43] T. Fagerberg, V. Zoete, S. Viatte, P. Baumgaertner, P. M. Alves, P. Romero et al., "Prediction of cross-recognition of peptide-HLA A2 by melan-a-specific cytotoxic T lymphocytes using threedimensional quantitative structure-activity relationships," PLoS ONE, vol. 8, no. 7, Article ID e65590, 2013.

[44] D. Barh, A. N. Misra, A. Kumar, and A. Vasco, "A novel strategy of epitope design in Neisseria gonorrhoeae," Bioinformation, vol. 5, no. 2, pp. 77-82, 2010.

[45] J. Bi, R. Song, H. Yang et al., "Stepwise identification of HLA$\mathrm{A}^{*}$ 0201-restricted $\mathrm{CD}^{+} \mathrm{T}$-cell epitope peptides from herpes simplex virus type 1 genome boosted by a steprank scheme," Biopolymers, vol. 96, no. 3, pp. 328-339, 2011.

[46] R. D. Bremel and E. J. Homan, "An integrated approach to epitope analysis II: a system for proteomic-scale prediction of immunological characteristics," Immunome Research, vol. 6, no. 1 , article 8, 2010.

[47] C. M. Diez-Rivero, B. Chenlo, P. Zuluaga, and P. A. Reche, "Quantitative modeling of peptide binding to TAP using support vector machine," Proteins, vol. 78, no. 1, pp. 63-72, 2010.

[48] E. Martínez-Naves, E. M. Lafuente, and P. A. Reche, "Recognition of the ligand-type specificity of classical and non-classical MHC I proteins," FEBS Letters, vol. 585, no. 21, pp. 3478-3484, 2011.

[49] M. Bhasin, E. L. Reinherz, and P. A. Reche, "Recognition and classification of histones using support vector machine," Journal of Computational Biology, vol. 13, no. 1, pp. 102-112, 2006.

[50] P. A. Reche and E. L. Reinherz, "PEPVAC: a web server for multi-epitope vaccine development based on the prediction of supertypic MHC ligands," Nucleic Acids Research, vol. 33, no. 2, pp. W138-W142, 2005.

[51] E. M. Lafuente and P. A. Reche, "Prediction of MHC-peptide binding: a systematic and comprehensive overview," Current Pharmaceutical Design, vol. 15, no. 28, pp. 3209-3220, 2009.

[52] A. Gaulton, L. J. Bellis, A. P. Bento et al. et al., "A large-scale bioactivity database for drug discovery," Nucleic Acids Research, pp. D1100-D1107, 2012.

[53] W. H. Cropper, Great Physicists: The Life and Times of Leading Physicists from Galileo to Hawking, Oxford University Press, New York, NY, USA, 2004.

[54] E. L. Bouzarth, A. Brooks, R. Camassa et al., "Epicyclic orbits in a viscous fluid about a precessing rod: theory and experiments at the micro-and macro-scales," Physical Review E, vol. 76, no. 1, part 2, Article ID 016313, 2007.

[55] M. Laberge, "Intrinsic protein electric fields: basic non-covalent interactions and relationship to protein-induced Stark effects," Biochimica et Biophysica Acta, vol. 1386, no. 2, pp. 305-330, 1998.

[56] F. M. Fernandez and J. A. Morales, "Perturbation theory without wave functions for the Zeeman effect in hydrogen," Physical Review A, vol. 46, no. 1, pp. 318-326, 1992.

[57] B. D. Marshall and W. G. Chapman, "Thermodynamic perturbation theory for associating fluids with small bond angles: effects of steric hindrance, ring formation, and double bonding," Physical Reiew E, vol. 87, no. 5, Article ID 052307, 12 pages, 2013.

[58] H. Gonzalez-Diaz, S. Arrasate, A. Gomez-San Juan et al., "New theory for multiple input-output perturbations in complex molecular systems. 1. Linear QSPR electronegativity models in physical, organic, and medicinal chemistry," Current Topics in Medicinal Chemistry, 2013.

[59] H. Gonzalez-Diaz, S. Arrasate, N. Sotomayor et al., "MIANN models in medicinal, physical and organic chemistry," Current Topics in Medicinal Chemistry, vol. 13, no. 5, pp. 619-641, 2013.
[60] G. E. P. Box and G. M. Jenkins, Time Series Analysis: Forecasting and Control, Holden-Day, San Francisco, Calif, USA, 1970.

[61] A. Speck-Planche, V. V. Kleandrova, F. Luan, and M. N. Cordeiro, "Unified multi-target approach for the rational in silico design of anti-bladder cancer agents," Anti-Cancer Agents in Medicial Chemistry, vol. 13, no. 5, pp. 791-800, 2013.

[62] A. Speck-Planche, V. V. Kleandrova, and M. N. Cordeiro, "New insights toward the discovery of antibacterial agents: multitasking QSBER model for the simultaneous prediction of antituberculosis activity and toxicological profiles of drugs," The European Journal of Pharmaceutical Sciences, vol. 48, no. 4-5, pp. 812-818, 2013.

[63] A. Speck-Planche, V. V. Kleandrova, F. Luan, and M. N. Cordeiro, "Multi-target inhibitors for proteins associated with Alzheimer: in silico discovery using fragment-based descriptors," Current Alzheimer Research, vol. 10, no. 2, pp. 117-124, 2013.

[64] A. Speck-Planche, V. V. Kleandrova, F. Luan, and M. N. Cordeiro, "In silico discovery and virtual screening of multitarget inhibitors for proteins in Mycobacterium tuberculosis," Combinatorial Chemistry \& High Throughput Screening, vol. 15, no. 8, pp. 666-673, 2012.

[65] A. Speck-Planche, V. V. Kleandrova, F. Luan, and M. N. D. S. Cordeiro, "Chemoinformatics in anti-cancer chemotherapy: multi-target QSAR model for the in silico discovery of antibreast cancer agents," The European Journal of Pharmaceutical Sciences, vol. 47, no. 1, pp. 273-279, 2012. 


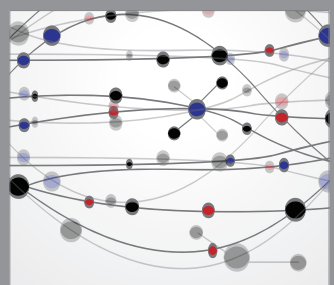

The Scientific World Journal
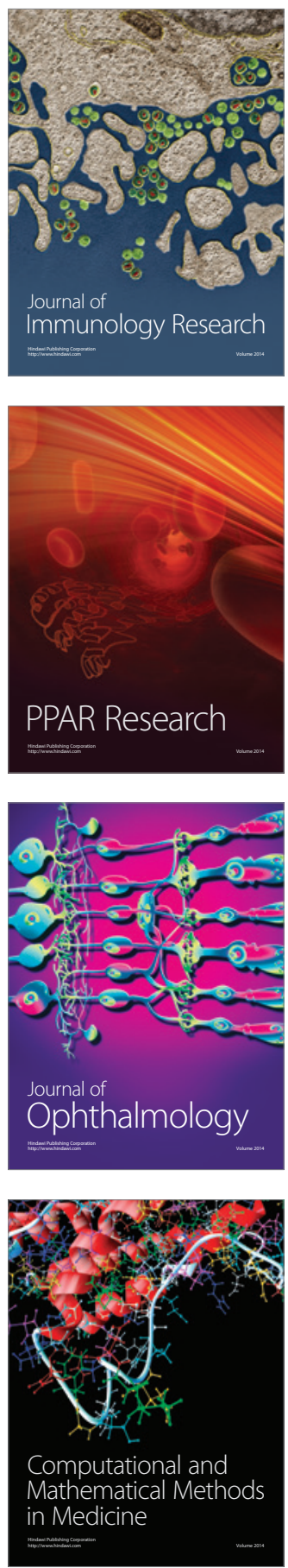

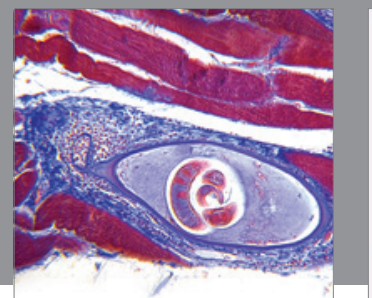

Gastroenterology

Research and Practice
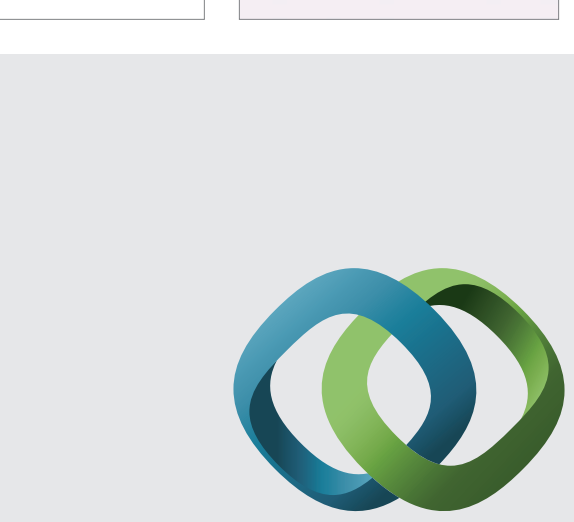

\section{Hindawi}

Submit your manuscripts at

http://www.hindawi.com
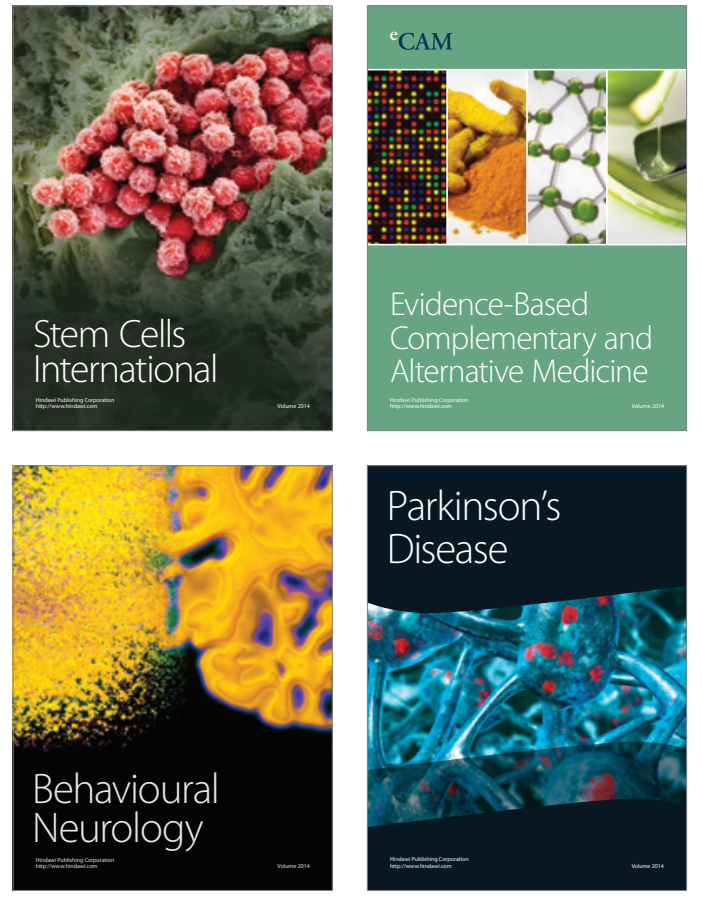
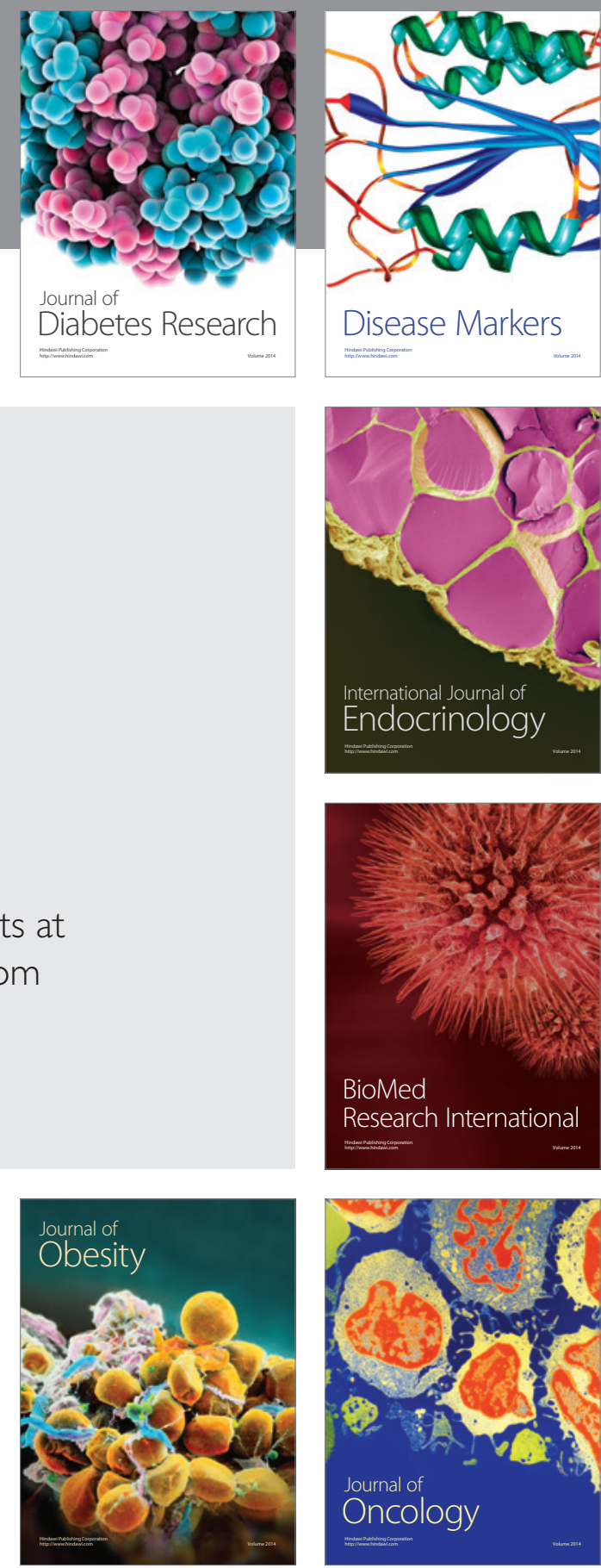

Disease Markers
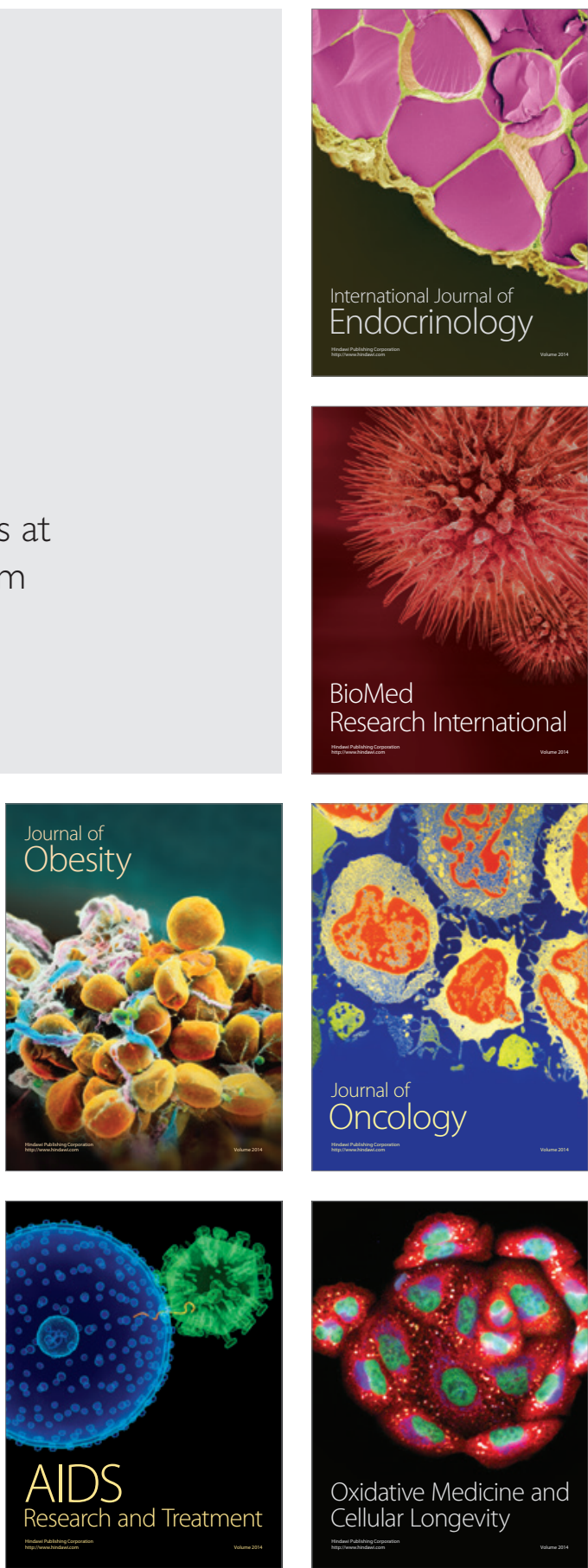